TRANSACTIONS OF THE

AMERICAN MATHEMATICAL SOCIETY

Volume 356, Number 6, Pages 2325-2348

S 0002-9947(03)03368-3

Article electronically published on November 4, 2003

\title{
UNITS IN SOME FAMILIES OF ALGEBRAIC NUMBER FIELDS
}

\author{
L. YA. VULAKH
}

\begin{abstract}
Multi-dimensional continued fractions associated with $G L_{n}(\mathbf{Z})$ are introduced and applied to find systems of fundamental units in some families of totally real fields and fields with signature $(2,1)$.
\end{abstract}

\section{INTRODUCTION}

Let $F$ be an algebraic number field of degree $n$. There exist exactly $n$ field embeddings of $F$ in $\mathbf{C}$. Let $s$ be the number of embeddings of $F$ whose images lie in $\mathbf{R}$, and let $2 t$ be the number of non-real complex embeddings so that $n=s+2 t$. The pair $(s, t)$ is said to be the signature of $F$. Let $\mathbf{Z}_{F}$ be the ring of integers of the field $F$. A unit in $F$ is an invertible element of $\mathbf{Z}_{F}$. The set of units in $F$ forms a multiplicative group which will be denoted by $\mathbf{Z}_{F}^{\times}$. In 1840 P. G. Lejeune-Dirichlet determined the structure of the group $\mathbf{Z}_{F}^{\times}$. He showed that $\mathbf{Z}_{F}^{\times}$is a finitely generated Abelian group of rank $r=s+t-1$, i.e. $\mathbf{Z}_{F}^{\times}$is isomorphic to $\mu_{F} \times \mathbf{Z}^{r}$, where $\mu_{F}$ is a finite cyclic group. $\mu_{F}$ is called the torsion subgroup of $\mathbf{Z}_{F}^{\times}$. Thus, there exist units $\epsilon_{1}, \ldots, \epsilon_{r}$ such that every element of $\mathbf{Z}_{F}^{\times}$can be written in a unique way as $\zeta \epsilon_{1}^{n_{1}} \ldots \epsilon_{r}^{n_{r}}$, where $n_{i} \in \mathbf{Z}$ and $\zeta$ is a root of unity in $F$. Such a set $\left\{\epsilon_{1}, \ldots, \epsilon_{r}\right\}$ is called a system of fundamental units of $F$. Finding a system of fundamental units of $F$ is one of the main computational problem of algebraic number theory (see e.g. 6], p. 217). Much work has been done to solve this problem for certain classes of algebraic number fields (see e.g. [22]). In the case of the real quadratic fields, the continued fraction algorithm provides a very efficient method for solving this problem (see e.g. [22], p. 119). This approach goes back to L. Euler, who applied continued fractions to solve Pell's equation $x^{2}-d y^{2}= \pm 1$. (If a square-free positive integer $d \equiv 2$ or $3 \bmod 4$ and $x, y$ is an integral solution of this equation, then $x+\sqrt{d} y$ is a unit in the real quadratic field $\mathbf{Q}(\sqrt{d})$. Moreover, any unit in $\mathbf{Q}(\sqrt{d})$ can be obtained this way.) Many attempts have been made to develop a similar algorithm that would find a system of fundamental units in other algebraic number fields. In the case of a cubic field, one of the most successful such algorithms was introduced by G. F. Voronoi [28]. A review of the multi-dimensional continued fraction algorithms and their properties that were known by 1980 can be found in [2].

In [30] and [31], a continued fraction algorithm associated with a discrete group acting in a hyperbolic space was defined. The purpose of the present paper is to extend this definition to the case of the group $\Gamma=\mathrm{GL}_{n}(\mathbf{Z}) /\{ \pm 1\}$ acting on

Received by the editors April 8, 2002 and, in revised form, March 30, 2003.

2000 Mathematics Subject Classification. Primary 11R27, 11J70.

Key words and phrases. Fundamental units, continued fractions, symmetric space.

The author was supported in part by NSA Grant MDA904-99-1-0052. 
$\mathcal{P}=S L_{n}(\mathbf{R}) / S O_{n}(\mathbf{R})$ and apply it to the problem of finding a system of fundamental units in an algebraic number field $F$. The symmetric space $\mathcal{P}$ can be identified with the set of definite quadratic forms in $n$ real variables with the leading coefficient one. $\mathcal{P}$ with the metric $d s^{2}=\operatorname{trace}\left(\left(X^{-1} d X\right)^{2}\right)$, where $X=\left(x_{i j}\right) \in \mathcal{P}$ and $d X=\left(d x_{i j}\right)$, is a Riemannian manifold (see e.g. [13] or [27]).

Assume that $g \in G L_{n}(\mathbf{R})$. Let $g a_{i}=\lambda_{i} a_{i}, i=1, \ldots, n$, so that $a_{i}$ is an eigenvector of $g$ corresponding to its eigenvalue $\lambda_{i}$. For simplicity, assume that all the eigenvalues of $g$ are distinct. Let $P=\left(a_{1}, \ldots, a_{n}\right)$ be the matrix with columns $a_{1}, \ldots, a_{n}$. The set of points in $\mathcal{P}$ fixed by $g$ will be called the axis $L_{P}$ of $g$. The axis $L_{P}$ of $g$ depends only on eigenvectors of $g$, i.e. on $P$, but not on its eigenvalues (see Section 3). $L_{P}$ is a totally geodesic submanifold of $\mathcal{P}$ of dimension $s+t-1$, where $s$ is the number of real and $2 t$ the number of non-real complex eigenvalues of $g$, so that $n=s+2 t$. If $t=0$, then $L_{P}$ is an $(n-1)$-flat in $\mathcal{P}$ (see e.g. [13]).

Let $\left(1, \omega_{2}, \ldots, \omega_{n}\right)$ be a $\mathbf{Z}$-basis of the ring of integers $\mathbf{Z}_{F}$ of a number field $F$ of degree $n$. Let $a_{1}=\left(1, \omega_{2}, \ldots, \omega_{n}\right)^{T}$. Let $\gamma \in \mathbf{Z}_{F}$. Then $\gamma \omega_{i}=\sum m_{i j} \omega_{j}$ or $\gamma a_{1}=M_{\gamma} a_{1}$, where $\omega_{1}=1, m_{i j} \in \mathbf{Z}$ and $M_{\gamma}=\left(m_{i j}\right)$ is a square matrix of order $n$. Let $\sigma_{i}$ be the $n$ distinct embeddings of $F$ in C. Let $a_{i}=\sigma_{i}\left(a_{1}\right)$ and $\gamma_{i}=\sigma_{i}(\gamma)$, where $\gamma_{1}=\gamma$. Then $\gamma_{i} a_{i}=M_{\gamma} a_{i}$ for $i=1, \ldots, n$. Thus, $a_{i}$ is an eigenvector of $M_{\gamma}$ corresponding to its eigenvalue $\gamma_{i}$. It is clear that the map $\gamma \longmapsto M_{\gamma}$ is an isomorphism of the ring of integers $\mathbf{Z}_{F}$ and the commutative ring of $\mathbf{Z}$-integral square matrices of order $n$ with the common axis $L_{P}$. The norm of $\gamma$ equals $\operatorname{det}\left(M_{\gamma}\right)$, so that $\gamma$ is a unit in $\mathbf{Z}_{F}$ if and only if $M_{\gamma} \in \mathrm{GL}_{n}(\mathbf{Z})$. The torsion-free subgroup $\Gamma_{L}$ of the stabilizer of $L_{P}$ is isomorphic to $\mathbf{Z}_{F}^{\times} / \mu_{F}$. Thus, the problem of finding a system of fundamental units of $F$ is equivalent to the problem of finding a set of generators of $\Gamma_{L}$. The [multi-dimensional continued fraction] Algorithm II introduced in this paper can be used to solve the latter problem. Here, a set of generators of $\Gamma_{L}$ and therefore a system of fundamental units is found in some families of fields $F$ of degree $n \leq 4$.

In Section 2, the notion of the height of a point in $\mathcal{P}$ is introduced. Let $w=$ $(1,0, \ldots, 0)^{T}$ and $W=w w^{T}$. In what follows, the point $W$, which belongs to the boundary of $\mathcal{P}$, is analogous to the point $\infty$ in the upper half-space model $H^{n+1}=$ $\left\{(z, t): z \in \mathbf{R}^{n}, t>0\right\}$ of the $(n+1)$-dimensional hyperbolic space (see [30] and [31]). The set $K=K(w)$ in $\mathcal{P}$ is defined so that, for every point $X \in \mathcal{P}$, the points in the $\Gamma$-orbit of $X$ with the largest height belong to $K(w)$. The images $K[g]$ of $K$, $g \in \Gamma$, under the action of $\Gamma$ form the $K$-tessellation of $\mathcal{P}$. The $K$-tessellation of $\mathcal{P}$ is $\Gamma$-invariant.

If $L_{P} \cap K[g] \neq \varnothing, g \in \Gamma$, then the vector $u=g^{-1} w \in \mathbf{Z}^{n}$ is called a convergent of $L_{P}$. In Section 3, it is shown that if $u$ is a convergent of $L_{P}$, then $\left|\left\langle a_{1}, u\right\rangle \ldots\left\langle a_{n}, u\right\rangle / \operatorname{det} P\right|$, where $\langle$,$\rangle denotes the dot product in \mathbf{R}^{n}$, is small (Theorem 7). Algorithm II, which is introduced in Section 3, can be applied to find the sets $R\left(g^{-1} w\right)=L_{P} \cap K[g] \neq \varnothing$, which form a tessellation of $L_{P}$, and the set of convergents of $L_{P}$.

If $g \in \Gamma$, then there are only finitely many sets $R(u)$ which are not congruent modulo the action of $\Gamma$. The union of non-congruent sets $R(u)$ forms a fundamental domain of $\Gamma_{L}$ in $L_{P}$. Assume that the characteristic polynomial $p(x)$ of $g$ is irreducible. Let $p(\epsilon)=0$. In Section 4 , the problem of finding a system of fundamental units in $F$ is solved for some families of totally real fields $F=\mathbf{Q}(\epsilon)$ by reducing it, as explained above, to the problem of finding a set of generators of $\Gamma_{L}$. In Examples 
1-4, new proofs of certain known results are given. The new results obtained in Examples 5 and 6 can be presented as follows.

Theorem 1. Assume that $t>3$ is a positive integer. Let $f(x)=x^{4}+t x^{3}-x^{2}-$ $t x+\alpha=x\left(x^{2}-1\right)(x+t)+\alpha, \alpha= \pm 1$. Let $f(\epsilon)=0$. Assume that $\left\{1, \epsilon, \epsilon^{2}, \epsilon^{3}\right\}$ is a $\mathbf{Z}$-basis of the maximal order $\mathbf{Z}_{F}$ of the totally real quadric field $F=\mathbf{Q}(\epsilon)$. Then $\mathbf{Z}_{F}^{\times} /\{ \pm 1\}=\langle\epsilon-1, \epsilon, \epsilon+1\rangle$.

Note that the Galois group of $F$ is $D_{4}$ if $\alpha=1$, and it is $S_{4}$ if $\alpha=-1$.

In Section 5 , the problem of finding a system of fundamental units is solved for some families of fields with signature $(2,1)$. The following theorems are proved in Examples 7, 8, and 9 respectively.

Theorem 2. Let $f(x)=x^{4}+t x^{3}+x^{2}+t x+1=x\left(x^{2}+1\right)(x+t)+1$, where $t \in \mathbf{Z}$. Let $f(\epsilon)=0$. Assume that $\eta=\epsilon+\epsilon^{-1}=\left(-t \pm \sqrt{t^{2}+4}\right) / 2$ is a fundamental unit of the quadratic subfield $K=\mathbf{Q}(\sqrt{d}), d=t^{2}+4$, of the dihedral quartic field $F=\mathbf{Q}(\epsilon)$ with signature $(2,1)$. Assume that $4 t^{2}-9$ is square-free. Then $\left\{1, \epsilon, \epsilon^{2}, \epsilon^{3}\right\}$ is a $\mathbf{Z}$-basis of the maximal order $\mathbf{Z}_{F}$ of the field $F$, and $\mathbf{Z}_{F}^{\times} /\{ \pm 1\}=\langle\epsilon, \eta\rangle$.

Theorem 3 (cf. [20]). Let $t \geq 4$ be an integer. Let $f(x)=x^{4}+t x^{3}+x^{2}+t x-1=$ $x\left(x^{2}+1\right)(x+t)-1$. Let $\epsilon$ be a real root of $f(x)$. Assume that the discriminant of the quartic field $F=\mathbf{Q}(\epsilon)$ with signature $(2,1)$ is square-free. Then $\left\{1, \epsilon, \epsilon^{2}, \epsilon^{3}\right\}$ is a $\mathbf{Z}$-basis of the maximal order $\mathbf{Z}_{F}$ of the field $F$, and $\mathbf{Z}_{F}^{\times} /\{ \pm 1\}=\langle\epsilon, \epsilon+t\rangle$.

Theorem 4. Let $\alpha= \pm 1$. Let $t \geq s$ be positive integers and let $\eta=\frac{1}{2}(t+\sqrt{d})$, where $d=t^{2}+4 \alpha$. Let $f(x)=f_{1}(x) f_{2}(x)=x^{4}+s t x^{3}+\left(t-\alpha s^{2}\right) x^{2}+s\left(t^{2}+2 \alpha\right) x-\alpha$, where $f_{1}(x)=\left(x^{2}+s \eta x-1 / \eta\right), f_{2}(x)=\left(x^{2}-s x / \eta+\eta\right)$. Let $f_{1}(\epsilon)=0$. Then $\eta \in F=\mathbf{Q}(\epsilon)$. Assume that $\eta$ is a fundamental unit of the quadratic subfield $K=\mathbf{Q}(\sqrt{d})$ of $F$ and that $\Delta=4 s^{2} t^{3}+12 \alpha s^{2} t-s^{4}+16 \alpha$ is square-free. Denote $p(x)=\left(s+\left(\alpha t-s^{2}\right) x+\alpha x^{3}\right) /\left(t s^{2}+1\right)$. Then $\left\{1, \epsilon, \epsilon^{2}, p(\epsilon)\right\}$ is a $\mathbf{Z}$-basis of the maximal order $\mathbf{Z}_{F}$ of the field $F$, the discriminant of $F$ is $d^{2} \Delta$, and $\mathbf{Z}_{F}^{\times} /\{ \pm 1\}=$ $\langle\epsilon, \eta\rangle$.

Families of cyclic quartic fields are considered in [15] and [35]. In [33], a onedimensional version of Algorithm II is applied to find fundamental units in a twoparameter family of complex cubic fields.

The author thanks the referee for the useful suggestions which led to a significant improvement of this work.

\section{Fundamental DOMAins AND $K$-TESSELlation}

Let $n \geq 2$ be a positive integer. Let $V_{n}$ be the space of symmetric $n \times n$ real matrices. The dimension of $V_{n}$ is $N=n(n+1) / 2$. The action of $g \in G=G L(n, \mathbf{R})$ on $X \in V_{n}$ is given by

$$
X \longmapsto X[g]=g^{T} X g .
$$

For a subset $S$ of $V_{n}$, denote $S[g]=\left\{X[g] \in V_{n}: X \in S\right\}$.

The one-dimensional subspaces of $V_{n}$ form the real projective space $V$ of dimension $N-1$, so that for any fixed nonzero $X \in V_{n}$, all the vectors $k X \in V_{n}$, $0 \neq k \in \mathbf{R}$, represent one point in $V$. Denote by $\mathcal{P} \subset V$ the set of (positive) definite elements of $V$ and by $\mathcal{B}$ the boundary of $\mathcal{P}(\mathcal{B}$ can be identified with the non-negative elements of $V$ of rank less than $n$ ). The group $G$ preserves both $\mathcal{P}$ and $\mathcal{B}$, as does its arithmetic subgroup $G L(n, \mathbf{Z})$. 
The space $V_{n}$ (and $V$ ) can be also identified with the set of quadratic forms $A[x]=x^{T} A x, A \in V_{n}, x \in \mathbf{R}^{n}$. With each point $a=\left(a_{1}, \ldots, a_{n}\right)^{T} \in \mathbf{R}^{n}$, we associate the matrix $A=a a^{T} \in \mathcal{B}$ and the quadratic form

$$
A[x]=\langle a, x\rangle^{2}=\left(a_{1} x_{1}+\ldots+a_{n} x_{n}\right)^{2}
$$

of rank one. For $g \in G$, we have $\langle g a, x\rangle=a^{T} g^{T} x=\left\langle a, g^{T} x\right\rangle$.

Let $w=(1,0, \ldots, 0)^{T}$ and $W=w w^{T}$. Then $\langle w, x\rangle^{2}=x_{1}^{2}$ and $W[g]=U=u u^{T}$, where $u=g^{T} w$.

Denote by $G_{\infty}$ and $\Gamma_{\infty}$ the stabilizers of $w$ in $G$ and $\Gamma=G L(n, \mathbf{Z}) /\{ \pm 1\}$ respectively. Then

$$
G_{\infty}=\{g \in G: g w=w\}=\left\{g \in G: g_{1}=w\right\},
$$

where $g_{1}$ is the first column of $g$. Thus, $g \in G_{\infty}$ if and only if $W\left[g^{T}\right]=W$.

We shall say that $A \in V$ is extremal if $|A[x]| \geq|A[w]|=a_{11}^{2}$ for any $x \in \mathbf{Z}^{n}$, $x \neq(0, \ldots, 0)$. Let $\mathcal{A}_{n}=\{X \in V: X[w] \neq 0\}$. It is clear that $\mathcal{P} \subset \mathcal{A}_{n}$. For $X \in \mathcal{A}_{n}$, we shall say that

$$
\operatorname{ht}(X)=|\operatorname{det}(X)|^{1 / n} /|X[w]|
$$

is the height of $X$ and, for a subset $S$ of $V$, we define the height of $S$ as

$$
\operatorname{ht}(S)=\operatorname{maxht}(X), \quad X \in S .
$$

The elements of $\mathcal{A}_{n}$ will be normalized so that $X[w]=1$. For a fixed $g \in \Gamma$, the set $\left\{X \in \mathcal{A}_{n}:|X[g w]|<1\right\}$ is called the $g$-strip (cf. [32], [29], where this definition is introduced for $n=2)$. It is clear that the $g h$-strip coincides with the $g$-strip for any $h \in \Gamma_{\infty}$. The plane

$$
L^{+}(g w)=L^{+}(g)=\left\{X \in \mathcal{A}_{n}: X[g w]=1\right\}
$$

is the boundary of the $g$-strip which cuts $\mathcal{P}$. Let $\mathcal{R}_{w}$ be the set of all extremal points of $V$. Denote

$$
K=K(w)=\mathcal{P} \cap \mathcal{R}_{w} .
$$

(In the notation of [1], p.148, $K$ is the dual core of $K_{\text {perf }}$.) Note that $K \subset \mathcal{A}_{n}$ is bounded by the planes $L^{+}(g)$. If $h \in \Gamma_{\infty}$, then $X[h w]=X[w]$ and, therefore, $\operatorname{ht}(X[h])=\operatorname{ht}(X)$. Thus,

$$
K[h]=K, \quad h \in \Gamma_{\infty} .
$$

Let $q(x)$ be an indefinite quadratic form in $n>2$ variables. By Margulis' theorem 19], if $q(x)$ is not a multiple of a quadratic form with integer coefficients, then the infimum of $|q(x)|$ taken over all $x \in \mathbf{Z}^{n}, x \neq(0, \ldots, 0)$, is equal to zero. Hence, all the points of $\mathcal{R}_{w}-\mathcal{P}$ are rational if $n>2$. By Meyer's theorem (see e.g. [5]), if the coefficients of $q(x)$ are rational and $n=5$, then there is $x_{0} \in \mathbf{Z}^{5}, x_{0} \neq(0, \ldots, 0)$, such that $q\left(x_{0}\right)=0$. Thus, $\mathcal{R}_{w}-\mathcal{P}$ is empty and $K=\mathcal{R}_{w}$ if $n>4$.

Let $D$ be any of the fundamental domains of $\Gamma$ obtained by Minkowski, Korkine and Zolotarev (see e.g. [24], p.13), or Grenier [16]. For $X \in D$ we have $X[w]=$ $\inf X[g w], g \in \Gamma$, in any of these cases. Hence, $\bigcup D[g]=K$, the union being taken over all $g \in \Gamma_{\infty}$. Note that the fundamental domain described in [16] coincides with the domain found by Korkine and Zolotarev in 1873 (see [18] or [24]). Unless a point $X \in \mathcal{P}$ is integral, like point $I$ in Example 1 below, in order to prove that $X$ is extremal, we shall show that $X[h]$ is Minkowski reduced for some $h \in \Gamma_{\infty}$. 
The main features of our approach to the problem of finding a system of fundamental units in an algebraic number field can be seen in the following simple example.

Example 1. Let $\Gamma=G L_{2}(\mathbf{Z})$. Let $X=\left(x_{i j}\right) \in V$. A point $X$ lies in $\mathcal{B}$ if and only if $\operatorname{det}(X)=x_{11} x_{22}-x_{12}^{2}=0$. Thus, when $n=2, \mathcal{B}$ is a conic in the projective plane $V$, and $\mathcal{P}$, which consists of the points $X$ with $\operatorname{det}(X)>0$, is the Klein model of the hyperbolic plane. Let $f(x)=x^{2}-t x-1$, where $t \in \mathbf{Z}$. Let $f(\epsilon)=0$. Assume that either $t^{2}+4$ or $t^{2} / 4+1$ is a square-free integer. Then $\{1, \epsilon\}$ is a $\mathbf{Z}$-basis of the maximal order $\mathbf{Z}_{F}$ of the field $F=\mathbf{Q}(\epsilon)$. Let

$$
E=\left[\begin{array}{ll}
0 & 1 \\
1 & t
\end{array}\right]
$$

Then $f(x)$ is a characteristic polynomial of $E$; and $a_{1}=(1, \epsilon)^{T}$ and $a_{2}=(1,-1 / \epsilon)^{T}$ are eigenvectors of $E$ corresponding to its eigenvalues $\epsilon$ and $-1 / \epsilon$ respectively. Let $A_{i}=a_{i} a_{i}^{T}$. Let $L_{P}$ be the axis of $E$. Then $L_{P}$ is the interval $\mu A_{1}+(1-\mu) A_{2}$, $0<\mu<1$, which is a geodesic in $\mathcal{P}$. The identity matrix $I$ is the intersection of $L_{P}$ with $L^{+}(E)$, and the interval $(I, I[E])$ is a fundamental domain of $\Gamma_{L}$ on $L_{P}$. Thus, $\Gamma_{L}=\langle E\rangle$ and, therefore, $\mathbf{Z}_{F}^{\times} /\{ \pm 1\}=\langle\epsilon\rangle$.

The period length of the corresponding continued fraction is one (see Remark 2 at the end of Section 3). More examples of this type can be found in [30] and [31].

Similar families of algebraic number fields of degree three and four are considered in Sections 4 and 5 .

By (2), $K[h g]=K[g]$ for any $g \in \Gamma$ and $h \in \Gamma_{\infty}$. Thus, the sets $K[g]$ are parameterized by the classes $\Gamma_{\infty} \backslash \Gamma$ or by primitive vectors $u=g^{-1} h^{-1} w=g^{-1} w$, so that $\pm u$ represent the same $K[g]$. The sets $K[g], g \in \Gamma_{\infty} \backslash \Gamma$, form a tessellation of $\mathcal{P}$ which will be called the $K$-tessellation. It is clear that the $K$-tessellation of $\mathcal{P}$ is $\Gamma$-invariant. The vertices of $K$ are called the perfect forms (see e.g. [1] or [24]). The perfect forms are known for $n \leq 7$.

\section{Axes of elements of $G$}

Let $g \in G$. Let $g a_{i}=\lambda_{i} a_{i}, i=1, \ldots, n$, where, for simplicity, we assume that $\lambda_{i} \neq \lambda_{j}$ if $i \neq j$. Here $a_{i}$ is an eigenvector of $g$ corresponding to its eigenvalue $\lambda_{i}$. Assume that $\left\langle a_{i}, w\right\rangle \neq 0, i=1, \ldots, n$. Then we can choose $a_{i}$ so that

$$
\left\langle a_{i}, w\right\rangle=1, \quad i=1, \ldots, n .
$$

$g \in \Gamma$ is said to be irreducible if its characteristic polynomial is irreducible over $\mathbf{Q}$. If $g \in \Gamma$ is irreducible, then all its eigenvalues are distinct. Assume that $\lambda_{1}, \lambda_{2}, \ldots, \lambda_{s}$ are real and $\lambda_{s+1}, \lambda_{s+2}, \ldots, \lambda_{s+t}, \bar{\lambda}_{s+1}, \bar{\lambda}_{s+2}, \ldots, \bar{\lambda}_{s+t}$ are non-real complex eigenvalues of $g$, so that $n=s+2 t$. Let $\lambda_{k} \neq \pm 1$. Let

$$
P=\left(a_{1}, \ldots, a_{s}, a_{s+1}, \bar{a}_{s+1}, \ldots, a_{s+t}, \bar{a}_{s+t}\right)
$$

be the matrix with columns $a_{1}, \ldots, a_{s}, a_{s+1}, \bar{a}_{s+1}, \ldots, a_{s+t}, \bar{a}_{s+t}$ and let

$$
H=\operatorname{diag}\left(\lambda_{1}, \ldots, \lambda_{s}, \lambda_{s+1}, \bar{\lambda}_{s+1}, \ldots, \lambda_{s+t}, \bar{\lambda}_{s+t}\right) .
$$

Then $g=P H P^{-1}$. For $k>s$, let $a_{k}=\alpha_{k}+i \beta_{k}$, where $\alpha_{k}, \beta_{k} \in \mathbf{R}^{n}$. Then

$$
\operatorname{det} P=(2 i)^{t} \operatorname{det}\left(a_{1}, \ldots, a_{s}, \beta_{s+1}, \alpha_{s+1}, \ldots, \beta_{s+t}, \alpha_{s+t}\right) \text {. }
$$


The totally geodesic submanifold $L_{P}$ of $\mathcal{P}$ fixed by $g=P H P^{-1}$ will be called the axis of $g$. The dimension of $L_{P}$ is $s+t-1$. It can be identified with the set of quadratic forms in $\mathcal{A}_{n}$

$$
q[x]=\sum_{i=1}^{s} \mu_{i}\left\langle x, a_{i}\right\rangle^{2}+\sum_{i=1}^{t} \mu_{s+i}\left|\left\langle x, a_{s+i}\right\rangle\right|^{2}, \quad \mu_{i} \geq 0, \sum_{i=1}^{s+t} \mu_{i}=1 .
$$

or

$$
q[x]=\sum_{i=1}^{s} \mu_{i}\left\langle x, a_{i}\right\rangle^{2}+\sum_{i=1}^{t} \mu_{s+i}\left(\left\langle x, \alpha_{s+i}\right\rangle^{2}+\left\langle x, \beta_{s+i}\right\rangle^{2}\right) .
$$

Hence

$$
\operatorname{det} q=(-4)^{-t} \mu_{1} \ldots \mu_{s} \mu_{s+1}^{2} \ldots \mu_{s+t}^{2}(\operatorname{det} P)^{2} .
$$

It follows from (4) that $L_{P}$ is the axis of $h \in G$ if and only if $a_{i}, i=1, \ldots, n$, are eigenvectors of $h$. Hence, the axis of $g$ depends only on its set of eigenvectors, i.e. on $P$, but not on the eigenvalues of $g$. A point $q \in L_{P}$ can be also represented as

$$
q=\sum_{i=1}^{s+t} \mu_{i} A_{i}, \quad A_{i}=a_{i} a_{i}^{T}, i \leq s, \quad A_{s+i}=\alpha_{s+i} \alpha_{s+i}^{T}+\beta_{s+i} \beta_{s+i}^{T} .
$$

Thus, $L_{P}$ is the simplex with vertices $A_{i}, i=1, \ldots, s+t$. All the faces of $L_{P}$ belong to $\mathcal{B}$. Note that $L_{P}\left[g^{T}\right]=L_{P}$. The curve $q\left[g^{v}\right]=\left(\sum \mu_{i}\left|\lambda_{i}\right|^{2 v} A_{i}\right) / \sum \mu_{i}\left|\lambda_{i}\right|^{2 v}$ is a geodesic in $L_{P}$ through $q$. If $\left|\lambda_{1}\right|<\left|\lambda_{2}\right|<\ldots<\left|\lambda_{s+t}\right|$, then $q\left[g^{v}\right] \rightarrow A_{1}$ as the real parameter $v \rightarrow-\infty$, and $q\left[g^{v}\right] \rightarrow A_{s+t}$ as $v \rightarrow \infty$. Note that if $t=0$, then $L_{P}$ is an $(n-1)$-flat in $\mathcal{P}$ and the stabilizer of $L_{P}$ in $G$ is a maximal commutative subgroup of $G$ (see e.g. 13]).

Denote $K\left(g^{-1} w\right)=K[g]$ and

$$
R\left(g^{-1} w\right)=K[g] \cap L_{P} \neq \varnothing, \quad g \in \Gamma_{\infty} \backslash \Gamma .
$$

The sets $R(u), u=g^{-1} w$, form a tessellation of $L_{P}$, which is invariant modulo the action of $\Gamma$ since the $K$-tessellation of $\mathcal{P}$ is $\Gamma$-invariant. We say that this tessellation is periodic if there are only a finite number of non-congruent sets $R(u)$ modulo the action of $\operatorname{Stab}\left(L_{P}, \Gamma\right)$. In that case, the union of all non-congruent sets $R(u)$ is a fundamental domain of $\operatorname{Stab}\left(L_{P}, \Gamma\right)$. The number of non-congruent sets $R(u)$ in the tessellation of $L_{P}$ will be called the period length.

Denote

$$
\gamma_{n}=1 / h_{n}, \quad h_{n}=\inf \operatorname{ht}(A), \quad A \in K(w) .
$$

Hermite's constant $\gamma_{n}$ is known for $n \leq 8$ (see e.g. [4]). Let $C_{n}=\gamma_{n} / n$. Then

$$
\begin{gathered}
C_{2}=1 / \sqrt{3}, \quad C_{3}=2^{1 / 3} / 3, \quad C_{4}=1 / \sqrt{8}, \quad C_{5}=8^{1 / 5} / 5, \\
C_{6}=1 / 3^{7 / 6}, \quad C_{7}=64^{1 / 7} / 7, \quad C_{8}=1 / 4,
\end{gathered}
$$

and for large $n$ (see [8]),

Let

$$
\frac{1}{2 \pi e} \leq C_{n} \leq \frac{1.744}{2 \pi e}
$$

$$
N_{P}(x)=\left\langle x, a_{1}\right\rangle \ldots\left\langle x, a_{n}\right\rangle=\left\langle x, a_{1}\right\rangle \ldots\left\langle x, a_{s}\right\rangle\left|\left\langle x, a_{s+1}\right\rangle \ldots\left\langle x, a_{s+t}\right\rangle\right|^{2},
$$

where $\left\langle x, a_{i}\right\rangle=x^{T} a_{i}$. Define

$$
\nu\left(L_{P}\right)=\inf \left|\frac{N_{P}(g w)}{\operatorname{det} P}\right|,
$$


where the infimum is taken over all $g \in \Gamma$. It is clear that $\nu\left(L_{P}\right)=\nu\left(L_{M P}[h]\right)$ for any $h \in \Gamma$, and $M=\operatorname{diag}\left(\mu_{1}, \ldots, \mu_{s}, \mu_{s+1}, \bar{\mu}_{s+1}, \ldots, \mu_{s+t}, \bar{\mu}_{s+t}\right)$, where $\mu_{1}, \ldots, \mu_{s} \in \mathbf{R}$, $\mu_{s+1}, \ldots, \mu_{s+t} \in \mathbf{C}$ and $\mu_{1} \ldots \mu_{s+t} \neq 0$. The projective invariant $\nu\left(L_{P}\right)$ is well known in the geometry of numbers (see e.g. [4] or [17]). In particular, for $n=s=3$, it was shown by Davenport [9], [10], [11] that $\nu\left(L_{P}\right) \leq 1 / 7$, where the equality holds only if $g a_{i}=\left(1, \alpha_{i}, \alpha_{i}^{2}\right), i=1,2,3$, the $\alpha_{i}$ being the roots of $\alpha^{3}+\alpha^{2}-2 \alpha-1=0$, for some $g \in \Gamma$. For other matrices $P, \nu\left(L_{P}\right) \leq 1 / 9$. Swinnerton-Dyer 26 found the consequent seventeen values of $\nu\left(L_{P}\right)$.

A point $q_{m} \in L_{P}$ is said to be the summit of $L_{P}$ if $\left|\operatorname{det}\left(q_{m}\right)\right|=\max |\operatorname{det}(q)|$, the maximum being taken over all $q \in L_{P}$. It is clear that if $R=L_{P} \cap K(w) \neq \varnothing$, then $q_{m} \in R$.

Lemma 5. Let $L_{P}$ be the totally geodesic manifold fixed by $g \in G$ and defined by (4), where $g a_{i}=\lambda_{i} a_{i}$. Let $P=\left(a_{1}, \ldots, a_{n}\right)$ be the matrix with columns $a_{1}, \ldots, a_{n}$. Then

$$
q_{m}[x]=\frac{1}{n} \sum_{i=1}^{s}\left\langle x, a_{i}\right\rangle^{2}+\frac{2}{n} \sum_{i=1}^{t}\left|\left\langle x, a_{s+i}\right\rangle\right|^{2}
$$

is the summit of $L_{P}$,

and

$$
\operatorname{ht}\left(L_{P}\right)=\frac{1}{n}\left|\frac{\operatorname{det} P}{N_{P}(w)}\right|^{2 / n}
$$

$$
\nu\left(L_{P}\right)=\inf \left(n \operatorname{nht}\left(L_{P}[g]\right)\right)^{-n / 2}, g \in \Gamma .
$$

Proof. The maximum of $m=\mu_{1} \ldots \mu_{s} \mu_{s+1}^{2} \ldots \mu_{s+t}^{2}$ subject to $\mu_{i} \geq 0, \sum_{i=1}^{s+t} \mu_{i}=1$, is attained when $\mu_{k}=1 / n, k \leq s$, and $\mu_{k}=2 / n, k>s$. Thus, $\max m=4^{t} n^{-n}$, (6) holds, and $\max \operatorname{det}(q)=\operatorname{det}\left(q_{m}\right)=|\operatorname{det} P|^{2} n^{-n}$. By ( 3), $N_{P}(w)=q[w]=1$. Hence

$$
\operatorname{ht}\left(L_{P}\right)=\left|\operatorname{det}\left(q_{m}\right)\right|^{1 / n}=\frac{1}{n}\left|\frac{\operatorname{det} P}{N_{P}(w)}\right|^{2 / n},
$$

and $\nu\left(L_{P}\right)=\inf \left(n h t\left(g L_{P}\right)\right)^{-n / 2}, g \in \Gamma$, as required.

It follows that, for any $L_{P}, \operatorname{ht}(X)=|\operatorname{det}(X)|^{1 / n} \rightarrow 0$ as $X \in L_{P}$ approaches the boundary of $L_{P}$. Note that $n q_{m}[x]$ is the form $\operatorname{size}\left(M_{x}\right)$ from [6], p. 169 .

In Example 1 above, by Lemma $5, q_{m}=\left(A_{1}+A_{2}\right) / 2=(I+I[E]) / 2$ and $\operatorname{det}\left(q_{m}\right)=t^{2} / 4+1$ and $\operatorname{ht}\left(L_{P}\right)=\sqrt{t^{2} / 4+1}$.

Assume that $L_{P} \cap K(w)=\varnothing$. Let $q_{m}$ be the summit of $L_{P}$. Since $q_{m} \notin K(w)$, there is $g \in \Gamma$ such that $\operatorname{ht}\left(L_{P}[g]\right) \geq \operatorname{ht}\left(q_{m}[g]\right)>\operatorname{ht}\left(q_{m}\right)=\operatorname{ht}\left(L_{P}\right)$. We have obtained the following.

Lemma 6. Let $L_{P}$ be the totally geodesic manifold fixed by $g \in G$ and defined by (4), where $g a_{i}=\lambda_{i} a_{i}$. Then

$$
\nu\left(L_{P}\right)=\inf \left(n \operatorname{ht}\left(L_{P}\left[g_{j}\right]\right)\right)^{-n / 2}, \quad L_{P} \cap K\left(g_{j} w\right) \neq \varnothing, \quad g_{j} \in \Gamma .
$$

By Lemma 6,

$$
\nu\left(L_{P}\right)<\left(n h_{n}\right)^{-n / 2}=C_{n}^{n / 2} .
$$

For $n=2, \nu\left(L_{P}\right)<C_{2}=1 / \sqrt{3} \cdot \sup \nu\left(L_{P}\right)=1 / \sqrt{5}$ is a well-known approximation constant. It is attained only if $g a_{i}=(1,(1 \pm \sqrt{5}) / 2)$ for some $g \in \Gamma$. For other matrices $P, \nu\left(L_{P}\right) \leq 1 / \sqrt{8}$ (cf. Example 1 above, where, for $t=1$ and 2 , the 
discriminants of the fields $F$ are 5 and 8 ). The set of values of $\nu\left(L_{P}\right)>1 / 3$ is discrete and consists of numbers $\left(9-4 / m^{2}\right)^{-1 / 2}$, where $m$ runs through the set of all positive integers such that $\left(m, m_{1}, m_{2}\right)$ is a solution of the Diophantine equation $m^{2}+m_{1}^{2}+m_{2}^{2}=3 m m_{1} m_{2}$. This result was obtained by Markov in 1879 (see e.g. [17, Section. 43).

When $n=3$, we have $\nu\left(L_{P}\right)<\sqrt{2 / 27}=0.2722$. This estimate does not depend on the dimension of $L_{P}$. It was shown by Davenport [12] that $\nu\left(L_{P}\right) \leq 1 / \sqrt{23}=$ 0.2085 when $s=t=1$, where the equality holds only if $\mathrm{ga}_{i}=\left(1, \alpha_{i}, \alpha_{i}^{2}\right), i=1,2,3$, the $\alpha_{i}$ being the roots of $\alpha^{3}-\alpha-1=0$, for some $g \in \Gamma$. As mentioned above, $\nu\left(L_{P}\right) \leq 1 / 7=0.1429$ when $\operatorname{dim} L_{P}=2$. 5 ,

Assume that $L_{P} \cap K(g w) \neq \varnothing$, where $g \in \Gamma$. Since $L_{P}[g] \cap K(w) \neq \varnothing$, by Lemma

$$
\operatorname{ht}\left(L_{P}[g]\right)=\operatorname{ht}\left(L_{g^{T} P}\right)=\frac{1}{n}\left|\frac{\operatorname{det} P}{N_{g^{T} P}(w)}\right|^{2 / n}>h_{n}=1 / \gamma_{n} .
$$

But $N_{g^{T} P}(x)=\left\langle x, g^{T} a_{1}\right\rangle \ldots\left\langle x, g^{T} a_{n}\right\rangle=\left\langle g x, a_{1}\right\rangle \ldots\left\langle g x, a_{n}\right\rangle$. Hence $N_{g^{T} P}(w)=$ $N_{P}(g w)$.

A vector $g w \in \mathbf{Z}^{n}$ such that $L_{P} \cap K(g w) \neq \varnothing$ will be called a convergent of $L_{P}$. We have proved the following.

Theorem 7. If a vector $u$ is a convergent of $L_{P}$ (that is, if $\left.L_{P} \cap K(u) \neq \varnothing\right)$, then

$$
\left|N_{P}(u)\right|<C_{n}^{n / 2}|\operatorname{det} P|,
$$

where $C_{n}=\gamma_{n} / n$ and $\gamma_{n}$ is Hermite's constant. Hence if $L_{P}$ cuts infinitely many sets $K(u)$, then this inequality has infinitely many solutions in $u \in \mathbf{Z}^{n}$.

A component of the boundary of a set $R(u)$ of codimension one will be called a face of $R(u)$. We shall say that sets $R(u)$ and $R\left(u^{\prime}\right)$ are neighbors if they have a common face, and if the sets $R(u)$ and $R\left(u^{\prime}\right)$ are neighbors then the convergents $u$ and $u^{\prime}$ are neighbors. The following lemma can be used to find the faces of $R=L_{P} \cap K(w) \neq \varnothing$ (see e.g. Example 4 below).

Lemma 8. Let $L_{P}$ be the axis of $g \in G$. Assume that $R=L_{P} \cap K(w) \neq \varnothing$. Let $R_{i}=L_{P} \cap K\left(u_{i}\right), i=1,2, \ldots$, be the neighbors of $R$, so that $R$ and $R_{i}$ have $a$ common face $\phi_{i}$. Then $\phi_{i} \subset L^{+}\left(u_{i}\right)$.

Proof. Assume that $K(w)$ and $K(g w)$ have a common face and that $X \in \bar{K}(w) \cap$ $\bar{K}(g w)$. Then $X[g] \in \bar{K}(w)$. Hence $X[w]=X[g w]=1$ and $X \in L^{+}(g w)$. Thus, the common face of $K(w)$ and $K(g w)$ lies in $L^{+}(g w)$.

Algorithm II below can be used to enumerate all the sets $R(u)$ which form the tessellation of $L_{P}$. Our ability to apply this algorithm is based on the assumption that one can find all the faces of $R=L_{P} \cap K(w)$ for any $L_{P}$. Then all the faces of any $R_{i}=L_{P} \cap K\left(u_{i}\right) \neq \varnothing$, where $u_{i}=g_{i} w$ and $g_{i} \in \Gamma$, can be found since $R_{i}\left[g_{i}\right]=L_{P}\left[g_{i}\right] \cap K(w)$. It is clear that $g_{i}$ is not unique. Algorithm II will make the choice of $g_{i}$ more specific.

Let $D$ be a fundamental domain of $\Gamma$. The floor of $D$ consists of the faces of $D$ which do not contain the point $W$. Let $\mathcal{F}_{D}=\left\{\phi_{1}, \ldots, \phi_{m}\right\}$ be the set of faces in the floor of $D$. Let $\mathcal{S}_{D}=\left\{S_{1}, \ldots, S_{m}\right\}, S_{i} \in \Gamma$, where the face $\phi_{i} \subset L^{+}\left(S_{i}^{-1}\right)$. When $n=2$ or 3 , the floor of $D$ consists of only one face, and one can choose $S_{1}$ to be the reflection with respect to this face (see [33], p. 1310, and Example 1). 
As mentioned above, the tessellation of $L_{P}$ is invariant with respect to the action of $\Gamma$. Hence, we can assume that $L_{P} \cap K(w) \neq \varnothing$. Indeed, if $L_{P} \cap K(w)=\varnothing$, take a point $X \in L_{P}$ and find $h \in \Gamma$ such that $X[h] \in K(w)$. (Any of the reduction algorithms (see e.g. [8] for references) can be used to find such an $h$.) Then $L_{P}[h] \cap K(w) \neq \varnothing$.

Denote by $V_{L}$ the set $\{R(u)\}$, where the sets $R(u)$ form the tessellation of $L=$ $L_{P}$. There is a unique graph $\bar{G}_{L}=\left(V_{L}, \bar{E}_{L}\right)$ associated with $L$ whose set of vertices is $V_{L}$, and there is an edge $\left(R, R^{\prime}\right) \in \bar{E}_{L}$ if and only if $R, R^{\prime} \in V_{L}$ are neighbors.

Algorithm II. This algorithm finds a spanning tree $G_{L}=\left(V_{L}, E_{L}\right)$ of the graph $\bar{G}_{L}$. An edge $\left(R, R^{\prime}\right) \in E_{L}$ is labeled by $T \in \Gamma$ if $R=L \cap K[g]$ and $R^{\prime}=L \cap K[T g]$. If the dimension of $L$ is one, then $\bar{G}_{L}=G_{L}$.

Input. A simplex $L \subset \mathcal{P}$ with vertices at $A_{i} \in \mathcal{B}$, where $A_{i}=a_{i} a_{i}^{T}$ for $i=$ $1, \ldots, s, A_{s+i}=\alpha_{s+i} \alpha_{s+i}^{T}+\beta_{s+i} \beta_{s+i}^{T}$ for $i=1, \ldots, t, a_{i}, \alpha_{s+i}, \beta_{s+i} \in \mathbf{R}^{n}$, and $\operatorname{det}\left(a_{1}, \ldots, a_{s}, \beta_{s+1}, \alpha_{s+1}, \ldots, \beta_{s+t}, \alpha_{s+t}\right) \neq 0$.

$R_{0}=L \cap K(w) \neq \varnothing$.

Output. A tree $G_{L}=\left(V_{L}, E_{L}\right)$ where $V_{L}=\{R(u)\}$, where sets $R(u)$ form the tessellation of $L$.

Denote by $(\mathcal{V}, \mathcal{E})$ a subtree of $G_{L}$ where $\mathcal{V} \subset V_{L}$ and $\mathcal{E} \subset E_{L}$ are current sets of vertices and edges of $G_{L}$ found.

Let $\mathcal{L}$ be an ordered list of leaves of the subtree $(\mathcal{V}, \mathcal{E})$ of $G_{L}$.

Let $R \in \mathcal{L}$. Let $\left\{R_{k}, k=1,2, \ldots\right\}$ be the set of neighbors of $R$ such that $R_{k} \notin \mathcal{V}$. Denote by $\mathcal{N}(R)$ an ordered set of faces $\psi_{k}=\bar{R} \cap \bar{R}_{k}$.

The root of $G_{L}$ is $R_{0}=R(w)$.

$\mathcal{V}=\mathcal{L}=\left\{R_{0}\right\}$ and $\mathcal{E}=\{\varnothing\}$.

Step 1. Let $\psi_{k} \in \mathcal{N}\left(R_{0}\right)$. Find $U_{k} \in \Gamma_{\infty}$ such that $\psi_{k} \subset \phi_{k}\left[U_{k}\right]$ for some $\phi_{k} \in \mathcal{F}_{D}$. Let $L_{k}^{\prime}=L\left[U_{k}^{-1}\right]$.

Then the face $\psi_{k}^{\prime}=\psi_{k}\left[U_{k}^{-1}\right]$ of $R^{\prime}=L_{k}^{\prime} \cap K(w)$ lies in $\phi_{k}$.

Step 2. Let $\psi_{k}^{\prime} \subset \phi_{k} \subset L^{+}\left(S_{k}^{-1}\right)$, where $S_{k} \in \mathcal{S}_{D}$, be as in step 1. Denote $T_{k}=S_{k} U_{k}$.

(Then $L_{k}=L_{k}^{\prime}\left[S_{k}^{-1}\right]=L\left[T_{k}^{-1}\right]$ cuts $K(w)$, and $L=L_{k}\left[T_{k}\right]$.)

Add $R_{k}=L \cap K\left[T_{k}\right]$ to $\mathcal{V}$ and $\mathcal{L}$, and add the edge $\left(R_{0}, R_{k}\right)$ labeled by $T_{k}$ to $\mathcal{E}$.

Step 3. If $\mathcal{N}\left(R_{0}\right) \neq \varnothing$, then go to step 1. Otherwise, remove $R_{0}$ from $\mathcal{L}$. $R_{0}$.)

(When $R_{0}$ is removed from $\mathcal{L}, \mathcal{L}$ consists of all the neighbors of $R_{0}$ and $\mathcal{V}=L \cup$

Step 4. Let $R \in \mathcal{L}$. Let $\left(R_{0}, R_{1}, \ldots, R_{i-1}, R\right)$ be the path in $G_{L}$ from $R_{0}$ to $R$, whose edges $\left(R_{s-1}, R_{s}\right)$ are labeled by $T_{s} \in \Gamma, s=1, \ldots, i$, so that

$$
R_{s}=L \cap K\left[T_{s} \ldots T_{1}\right]
$$

and $R=L \cap K[g]$, where $g=T_{i} \ldots T_{1}$.

(Then $R\left[g^{-1}\right]=L\left[g^{-1}\right] \cap K(w) \neq \varnothing$. If $L\left[g^{-1}\right]=L$, then $R\left[g^{-1}\right]=R_{0}$ and $\left.g \in \Gamma_{L}.\right)$

Step 5. Let $\psi_{k} \in \mathcal{N}(R)$. Find $U_{k} \in \Gamma_{\infty}$ such that $\psi_{k}\left[g^{-1}\right] \subset \phi_{k}\left[U_{k}\right]$ for some $\phi_{k} \in \mathcal{F}_{D}$. Let $L_{k}^{\prime}=L\left[g^{-1} U_{k}^{-1}\right]$.

Then the face $\psi_{k}^{\prime}=\psi_{k}\left[g^{-1} U_{k}^{-1}\right]$ of $R^{\prime}=L_{k}^{\prime} \cap K(w)$ lies in $\phi_{k}$.

Step 6. Let $\psi_{k}^{\prime} \subset \phi_{k} \subset L^{+}\left(S_{k}^{-1}\right)$, where $S_{k} \in \mathcal{S}_{D}$, be as in step 6. Denote $T_{k}=S_{k} U_{k}$.

(Then $L_{k}=L_{k}^{\prime}\left[S_{k}^{-1}\right]=L\left[g^{-1} T_{k}^{-1}\right]$ cuts $K(w)$, and $L=L_{k}\left[T_{k} g\right]$.)

Add $R_{k}=L \cap K\left[T_{k} g\right]$ to $\mathcal{V}$ and $\mathcal{L}$, and add the edge $\left(R, R_{k}\right)$ labeled by $T_{k}$ to $\mathcal{E}$. 
Step 7. If $\mathcal{N}(R) \neq \varnothing$, then go to step 5. Otherwise, remove $R$ from $\mathcal{L}$ and go to step 4 .

(When $R$ is removed from $\mathcal{L}$, all the neighbors of $R$ have been added to $\mathcal{V}$.)

Remarks. 1. By (5) $), R=L \cap K[g]=R\left(g^{-1} w\right)$, and the convergent associated with the set $R$ is $u=g^{-1} w$, where $g=T_{i} \ldots T_{1}$. Thus, Algorithm II can be used to find all the convergents of $L$.

2. Assume that the sets $R_{i}$ form the tessellation of the axis $L_{P}$ of an irreducible $g \in \Gamma$. Then the number of non-congruent sets $R_{i}$ is finite and the tessellation of $L_{P}$ is periodic. Let $R_{1} \cup \ldots \cup R_{p}=D_{L}$ be a fundamental domain of $\Gamma_{L}=\left\langle E_{1}, \ldots, E_{s+t-1}\right\rangle$ in $L_{P}$. Let $V_{L}^{\prime}=\left\{u_{1}, \ldots, u_{p}\right\}$ be the set of all convergents of $L_{P}$ associated with the vertices $R_{1}, \ldots, R_{p} \in V_{L}$. Then the set of all convergents of $L_{P}$ is $\left\{g u: g \in \Gamma_{L}\right.$, $\left.u \in V_{L}^{\prime}\right\}$. In Example 1 above, the set of convergents of $L_{P}$ is $\left\{E^{n} w, n \in \mathbf{Z}\right\}$. In Example 4 below, $p=4$. In all other examples, $p=1$, i.e., it will be shown in each of these cases that $R(w)$ is a fundamental domain of $\Gamma_{L}$.

3 . If vertices $R_{k}$ on level $i$ of the tree $G_{L}$ have been added to $\mathcal{V}$ in step 6 , then all the vertices of $G_{L}$ on levels $<i$ belong to $\mathcal{V}$. If $L$ is the axis of an irreducible $g \in \Gamma$, then in a finite number of steps $\mathcal{V}$ will contain a complete set of non-congruent sets $R(u)$. Thus, Algorithm II finds a fundamental domain and a set of generators of the group $\Gamma_{L}$ in a finite number of steps.

4. For the axis $L_{P}$ defined by (44), it can be shown that $\left\langle u, a_{1}\right\rangle \rightarrow 0$ as $R(u) \rightarrow$ $A_{1}=a_{1} a_{1}^{T}$ (see 33, Lemma 9).

\section{UNITS IN TOTALLY REAL FIELDS}

Let $\left(1, \omega_{2}, \ldots, \omega_{n}\right)$ be a $\mathbf{Z}$-basis of the ring of integers $\mathbf{Z}_{F}$ of a number field $F$ of degree $n$. Let $a_{1}=\left(1, \omega_{2}, \ldots, \omega_{n}\right)^{T}$. Let $\gamma \in \mathbf{Z}_{F}$. Then $\gamma \omega_{i}=\sum m_{i j} \omega_{j}$ or $\gamma a_{1}=M_{\gamma} a_{1}$, where $\omega_{1}=1, m_{i j} \in \mathbf{Z}$ and $M_{\gamma}=\left(m_{i j}\right)$ is a square matrix of order $n$. As explained in Section 1, the map $\gamma \longmapsto M_{\gamma}$ is an isomorphism of the ring of integers $\mathbf{Z}_{F}$ and the commutative ring of $\mathbf{Z}$-integral square matrices of order $n$ with the common axis $L_{P}$ defined by (4), where $(s, t)$ is the signature of $F$. The norm of $\gamma$ equals $\operatorname{det}\left(M_{\gamma}\right)$, so that $\gamma$ is a unit in $\mathbf{Z}_{F}$ if and only if $M_{\gamma} \in \mathrm{GL}{ }_{n}(\mathbf{Z})$. The group of units in $\mathbf{Z}_{F}$ is not isomorphic to $\operatorname{Stab}\left(L_{P}, \Gamma\right)$. On the one hand, $\Gamma=\mathrm{GL}_{n}(\mathbf{Z}) /\{ \pm 1\}$. On the other hand, the group $\operatorname{Stab}\left(L_{P}, \Gamma\right)$ is not necessarily commutative, since there may exist a non-trivial homomorphism $\operatorname{Gal}(F) \rightarrow \operatorname{Stab}\left(L_{P}, \Gamma\right)$. However, the torsion-free subgroup $\Gamma_{L}$ of $\operatorname{Stab}\left(L_{P}, \Gamma\right)$ is isomorphic to $\mathbf{Z}_{F}^{\times} / \mu_{F}$. Thus, the problem of finding a system of fundamental units of $F$ is equivalent to the problem of finding a set of generators of $\Gamma_{L}$. If $s>0$, then the torsion group $\mu_{F}=\{ \pm 1\}$, and $\Gamma_{L}$ is isomorphic to $\mathbf{Z}_{F}^{\times} /\{ \pm 1\}$. Note that, by Lemma 5 ,

$$
\operatorname{ht}\left(L_{P}\right)=\frac{1}{n}|d(F)|^{1 / n},
$$

where $d(F)=\operatorname{det}^{2}(P)$ is the discriminant of $F$.

A point $X=\left(x_{i j}\right) \in \mathcal{P}$ is said to be rational over the field $K$ if all $x_{i j} \in K$. A subset $S$ of $\mathcal{P}$ is rational over $K$ if the set of rational points of $S$ is dense in $S$. It is clear that the summit $q_{m}$ (see (6) ) of $L_{P}$ is rational over some real subfield $F_{L}$ of the Galois closure of $F$. Let $\Gamma_{L}(\mathbf{Q})$ be the stabilizer of $L_{P}$ in $G L_{n}(\mathbf{Q})$. The $\Gamma_{L}(\mathbf{Q})$-orbit of $q_{m}$ is dense in $L_{P}$. Hence $L_{P}$ is rational over $F_{L}$. Assume that $\operatorname{dim}\left(L_{P}\right)=r$. Then any vertex of $R=L_{P} \cap K(w)$ is the intersection of $L_{P}$ with $r$ rational (over Q) planes $L^{+}\left(g_{i}\right)$. Hence all the vertices of $R$ are rational over $F_{L}$. In this section, 
$F$ is a totally real field, $\operatorname{dim}\left(L_{P}\right)=n-1$, and $F_{L}=\mathbf{Q}$. In Section 5 , the signature of $F$ is $(2,1)$.

Let $f(x)=x^{n}-c_{n-1} x^{n-1}-\ldots-c_{1} x-c_{0}$ be an irreducible polynomial with integral coefficients. Let $f(\delta)=0$. Let $F=\mathbf{Q}(\delta)$. Assume that $\mathbf{Z}_{F}$ has the power basis $\left\{1, \delta, \ldots, \delta^{n-1}\right\}$. Let $a_{1}=\left(1, \delta, \ldots, \delta^{n-1}\right)^{T}$. The integral matrix $C$ such that $C a_{1}=\delta a_{1}$ is said to be the companion matrix of $f(x)$. Let $a_{i}=\left(1, \delta_{i}, \ldots, \delta_{i}^{n-1}\right)^{T}$, $i=1,2, \ldots, n$, where $\delta_{1}=\delta$. An equation of the axis $L_{P}$ of $C^{T}$ is

$$
q=\sum \mu_{i} A_{i}, \quad A_{i}=\left(a_{k m}\right)=a_{i} a_{i}^{T}, \mu_{i}>0, \mu_{1}+\ldots+\mu_{n}=1,
$$

where $a_{k m}=\delta_{i}^{k+m-2}$. Thus a point $q=\left(q_{k m}\right)$ of $L_{P}$ can be identified with the vector $\left[p_{0}, p_{1}, p_{2}, \ldots, p_{2 n-2}\right]$, where $q_{k m}=p_{k+m-2}$ and

$$
p_{i}=\mu_{1} \delta_{1}^{i}+\ldots+\mu_{n} \delta_{n}^{i}, i=0,1, \ldots, n-1 .
$$

Since $\delta_{j}^{n}=c_{n-1} \delta_{j}^{n-1}+\ldots+c_{1} \delta_{j}+c_{0}$,

$$
p_{i}=c_{n-1} p_{i-1}+c_{n-2} p_{i-2}+\ldots+c_{1} p_{i-n+1}+c_{0} p_{i-n}, i=n, n+1, \ldots, 2 n-2 .
$$

Using $p_{0}, \ldots, p_{n-1}$ as parameters on $L_{P}$ instead of $\left\{\mu_{i}, i=1, \ldots, n\right\}$, we obtain the following.

Lemma 9. Assume that the ring of integers $\mathbf{Z}_{F}$ of a totally real field $F$ has a power basis. A point $q=\left[p_{0}, p_{1}, p_{2}, \ldots, p_{2 n-2}\right]$, defined as above, belongs to the plane spanned by $L_{P}$ if and only if (7) holds, in which case the point $q \in L_{P}$ is uniquely determined by its first row:

$$
q=\left[p_{0}, p_{1}, \ldots, p_{n-1}\right] .
$$

In Example 1 above, $I=[1,0]$ and $I[E]=[1, t]$ in these coordinates. In the examples below, we shall use these coordinates for a point $q \in L_{P}$ whenever the ring of integers $\mathbf{Z}_{F}$ of a totally real field $F$ has a power basis.

Example 2. Here we consider the case of the simplest cubic fields (see [25]). These are the cyclic fields of discriminant $\left(t^{2}+3 t+9\right)^{2}$. The field $F=\mathbf{Q}\left(\epsilon_{1}\right)$ is generated by a root $\epsilon_{1}$ of $f(x)=x^{3}-t x^{2}-(t+3) x-1$. Assume that $t^{2}+3 t+9$ is square-free. Then $\left\{1, \epsilon_{1}, \epsilon_{1}^{2}\right\}$ is a basis of $\mathbf{Z}_{F}$, units $\epsilon_{1}$ and $\epsilon_{2}=-1 /\left(1+\epsilon_{1}\right)$ both are the roots of this polynomial, and $\mathbf{Z}_{F}^{\times} /\{ \pm 1\}=\left\langle\epsilon_{1}, \epsilon_{2}\right\rangle$ (see [34]). For $t=-1,0$, and 1 , the discriminants of $F$ are $7^{2}, 9^{2}$, and $13^{2}$ respectively. These fields were considered by Davenport [9], 10], [11] (see also [3]), and Swinnerton-Dyer [26].

Let $E^{T}$ be the companion matrix of $f(x)$ and let $E_{1}=E+I$. Let $L_{P}$ be the axis of $E$. Then the torsion-free subgroup $\Gamma_{L}$ of the stabilizer of $L_{P}$ in $\Gamma$ is generated by $E$ and $E_{1}$. Let $E^{T} a_{i}=\epsilon_{i} a_{i}$ and $A_{i}=a_{i} a_{i}^{T}$, where $a_{i}=\left(1, \epsilon_{i}, \epsilon_{i}^{2}\right), i=1,2,3$. Then $q\left(\mu_{1}, \mu_{2}, \mu_{3}\right)=\mu_{1} A_{1}+\mu_{2} A_{2}+\mu_{3} A_{3}, \mu_{i}>0, \mu_{1}+\mu_{2}+\mu_{3}=1$, is an equation of $L_{P}$.

Let $R=L_{P} \cap K(w)$. An edge of $R$ is the intersection of $L_{P}$ with some $L^{+}(g)$, $g \in \Gamma$, which contains a face of $K(w)$, and the vertices of $R$ are the points of intersection of $L_{P}$ with some faces of $K(w)$ of codimension two. Denote $E_{2}=$ $E E_{1}^{-1}$. Let $F_{1}$ be the intersection of $L_{P}, L^{+}(E)$, and $L^{+}\left(E_{2}\right)$, and let $G_{1}$ be the intersection of $L_{P}, L^{+}(E)$, and $L^{+}\left(E_{1}\right)$. Since $\mathbf{Z}_{F}$ has a power basis, Lemma 9 is applicable. In our case, $R$ is the hexagon with vertices at $F_{1}=[1,0,1], F_{2}=F_{1}[E]$, $F_{3}=F_{1}\left[E_{2}\right], G_{1}=[1,-1 / 2,1], G_{2}=G_{1}\left[E_{1}\right], G_{3}=G_{1}[E]$ with $\operatorname{det}\left(F_{i}\right)=1$, $\operatorname{det}\left(G_{i}\right)=\left(t^{2}+3 t+9\right) / 8$. The sides of $R$ are identified as follows: $E: F_{1} G_{1} \rightarrow F_{2} G_{3}$; 
$E_{1}: F_{3} G_{1} \rightarrow F_{2} G_{2} ; E_{2}: F_{1} G_{2} \rightarrow F_{3} G_{3}$. Since the forms $F_{i}$ are integral, they are extremal.

Let $t=3 u+k$, where $|k| \leq 1$. Denote

$$
h=\left[\begin{array}{ccc}
1 & 0 & h_{13} \\
0 & 1 & u_{23} \\
0 & 0 & 1
\end{array}\right], \quad G_{0}=\left[\begin{array}{ccc}
1 & -1 / 2 & 0 \\
-1 / 2 & 1 & k / 2 \\
0 & k / 2 & g_{33}
\end{array}\right]
$$

where $h_{13}=-1-u, h_{23}=-2 u$, and $g_{33}=\left(4 \operatorname{det}\left(G_{1}\right)+k^{2}\right) / 3$. Then $G_{0}=G_{1}[h]$ is Minkowski reduced (8], pp. 396-397). Hence the points $G_{i}$ are extremal.

Hence $R=L_{P} \cap K(w)$ is a fundamental domain of $\left\langle E, E_{1}\right\rangle$, and therefore we have given a new proof that $\mathbf{Z}_{F}^{\times} /\{ \pm 1\}=\langle\epsilon, \epsilon+1\rangle$.

Note that if $F_{1}=q\left(\mu_{1}, \mu_{2}, \mu_{3}\right)$, then $F_{2}=q\left(\mu_{2}, \mu_{3}, \mu_{1}\right)$ and $F_{3}=q\left(\mu_{3}, \mu_{1}, \mu_{2}\right)$. The same relations hold for $G_{1}, G_{2}$, and $G_{3}$. Also, for the summit $q_{m}$ of $L_{P}$, we have

$$
q_{m}=\frac{1}{3} \sum A_{i}=\frac{1}{3} \sum F_{i}=\frac{1}{3} \sum G_{i} .
$$

Example 3. Let $t \geq 3$ be a positive integer. Let $f(x)=x^{3}+(t-1) x^{2}-t x-1$. Let $f(\epsilon)=0$. Assume that the discriminant of $f(x)$ is square-free. Then $\left\{1, \epsilon, \epsilon^{2}\right\}$ is a basis of $\mathbf{Z}_{F}$, where $F=\mathbf{Q}(\epsilon)$ is a totally real field. The field $F$ is non-Galois, and it is exceptional; that is, $\mathbf{Z}_{F}^{\times} /\{ \pm 1\}=\langle\epsilon, \epsilon-1\rangle$ (see e.g. [14], 21]). Since $\mathbf{Z}_{F}$ has a power basis, Lemma 9 is applicable.

Let $E^{T}$ be the companion matrix of $f(x), E_{1}=E-I$, and $E_{2}=E E_{1}^{-1}$. Let $L_{P}$ be the axis of $E$. Let $F_{1}$ be the intersection of $L_{P}, L^{+}(E)$, and $L^{+}\left(E_{1}\right)$, and let $G_{1}$ be the intersection of $L_{P}, L^{+}(E)$, and $L^{+}\left(E_{2}\right)$. Let $c=t^{2}+3 t+1$. The region $R=K(w) \cap L_{P}$ is the hexagon with vertices at $F_{1}=[1,1 / 2,1], F_{2}=F_{1}[E]$, $F_{3}=F_{1}\left[E_{1}\right], G_{1}=[1,-(2 t+4) / c, 1], G_{2}=G_{1}[E]$, and $G_{3}=G_{1}\left[E_{2}\right]$ with $\operatorname{det}\left(F_{i}\right)=$ $\left(t^{2}+3 t-9\right) / 8$ and $\operatorname{det}\left(G_{i}\right) \sim 1$ as $t \rightarrow \infty$. Let $h$ be as in Example 2 with $h_{13}=-u$, $h_{23}=-2+2 u$. Then $F_{1}[h]$ is Minkowski reduced. Hence the points $F_{i}, i=1,2,3$, are extremal. Denote

$$
h=\left[\begin{array}{lll}
1 & 0 & 1 \\
0 & 1 & t \\
0 & 0 & 1
\end{array}\right], \quad G_{0}=\left[\begin{array}{lll}
1 & -(2 t+4) / c & (2 t+2) / c \\
-(2 t+4) / c & 1 & -2 / c \\
(2 t+2) / c & -2 / c & 1
\end{array}\right] .
$$

The point $G_{0}=G_{1}[h]$ is Minkowski reduced ([8], pp. 396-397); hence the points $G_{i}, i=0,1,2,3$, are extremal. The sides of $R$ are identified as follows: $E: F_{1} G_{1} \rightarrow$ $G_{2} F_{2} ; E_{1}: F_{1} G_{3} \rightarrow F_{3} G_{2} ; E_{2}: F_{3} G_{1} \rightarrow F_{2} G_{3}$. Hence $R$ is a fundamental domain of $\left\langle E, E_{1}\right\rangle$, and therefore we have given a new proof that $\mathbf{Z}_{F}^{\times} /\{ \pm 1\}=\langle\epsilon, \epsilon-1\rangle$.

Note that now neither $\left(\sum F_{i}\right) / 3$ nor $\left(\sum G_{i}\right) / 3$ is the summit of $L_{P}$.

Example 4. Let $f(x)=x^{3}+(t+2) x^{2}+(2 t-1) x-1$, where $t \in \mathbf{Z}$. Let $f(\epsilon)=0$. Assume that the discriminant of $f(x)$ is square-free. Then $\left\{1, \epsilon, \epsilon^{2}\right\}$ is a basis of $\mathbf{Z}_{F}$, where $F=\mathbf{Q}(\epsilon)$ is a totally real field. The field $F$ is non-Galois, and $\mathbf{Z}_{F}^{\times} /\{ \pm 1\}=\langle\epsilon, \epsilon+2\rangle$ (see [21]). When $t=3$, the discriminant of the field is 148 . This case was considered by Swinnerton-Dyer [26] (see also [23]).

Let $E^{T}$ be the companion matrix of $f(x), E_{1}=E+2 I$, and $E_{2}=E E_{1}$. Let $L_{P}$ be the axis of $E$. Since $\mathbf{Z}_{F}$ has a power basis, Lemma 9 is applicable. 
Let $d=2 t^{2}-5 t+1$. Denote

$$
\begin{gathered}
F_{1}=[1,-1 / 2+1 /(4 t-6), 1], \quad F_{2}=F_{1}[E], \quad F_{3}=F_{1}\left[E_{2}\right], \\
G_{1}=[1,-1,2], \quad G_{2}=G_{1}\left[E_{2}\right], \\
H_{1}=[1,-3 / 2,3], \quad H_{2}=H_{1}\left[E_{1}\right], \\
K_{1}=[1,(2-t) / d, 1], \quad K_{2}=K_{1}[E], \\
M_{1}=[1,-1 /(2 t-1), 1], \quad M_{2}=M_{1}\left[E_{1}^{-1}\right], \quad M_{3}=M_{2}\left[E_{2}\right]
\end{gathered}
$$

with $\operatorname{det}\left(G_{1}\right)=1, \operatorname{det}\left(H_{1}\right)=(3 t+1) / 8, \operatorname{det}\left(M_{1}\right)=(6 t+1)(t-1)^{2} /(2 t-1)^{3}$, and $\operatorname{det}\left(F_{1}\right), \operatorname{det}\left(K_{1}\right) \sim 3 / 4$ as $t \rightarrow \infty$.

Denote $u_{1}=\left(\begin{array}{lll}0 & t & 1\end{array}\right)^{T}, u_{2}=\left(\begin{array}{lll}1 & 1 & 0\end{array}\right)^{T}, u_{3}=\left(\begin{array}{lll}t & 1 & 0\end{array}\right)^{T}$ and $h_{1}=\left[\begin{array}{ll}u_{1}, e_{1}, e_{2}\end{array}\right]$, $h_{i}=\left[u_{i}, e_{1}, e_{3}\right], i=2,3$, where $\left[e_{1}, e_{2}, e_{3}\right]$ is the identity matrix. Then $F_{1}=$ $L_{P} \cap L^{+}(E) \cap L^{+}\left(E_{2}\right), G_{1}=L_{P} \cap L^{+}\left(E_{2}\right) \cap L^{+}\left(u_{2}\right), H_{1}=L_{P} \cap L^{+}\left(E_{1}\right) \cap L^{+}\left(u_{2}\right)$, $K_{1}=L_{P} \cap L^{+}(E) \cap L^{+}\left(u_{1}\right)$ and $M_{1}=L_{P} \cap L^{+}(E) \cap L^{+}\left(E_{1}^{-1}\right)$.

The points $G_{1}$ and $H_{1}$ are extremal since they are integral. Let

$$
h(s)=\left[\begin{array}{lll}
1 & 0 & 0 \\
0 & 1 & s \\
0 & 0 & 1
\end{array}\right] .
$$

Then $F_{1}[h(2)]$ and $K_{1}[h(t)]$ are Minkowski reduced ([8], pp. 396-397). Hence $F_{i}$ and $K_{i}$ are also extremal. The points $M_{i}, i=1,2,3$, are not extremal. But the points $M_{i}^{\prime}=(2 t-1) /(2 t-2) M_{i}\left[h_{i}\right]$ are extremal with $\operatorname{det}\left(M_{i}^{\prime}\right)=(6 t+1) /(8 t-8)$. In particular, $M_{1}\left[h_{1}\right]$ is Minkowski reduced.

The fundamental domain of $\Gamma_{L}=\left\langle E, E_{1}\right\rangle$ is the hexagon $D_{L}=R \cup R_{1} \cup R_{2} \cup R_{3}$ with vertices at $F_{1}, M_{1}, F_{3}, M_{3}, F_{2}$ and $M_{2}$. The region $R=L_{P} \cap K(w)$ is the 9-gon with vertices at $F_{1}, K_{1}, H_{2}, F_{3}, G_{2}, K_{2}, F_{2}, H_{1}$ and $G_{1}$. Regions $R_{1}, R_{2}$ and $R_{3}$ are triangles. The vertices of $R_{1}$ are $M_{1}, K_{1}$ and $H_{2}$, of $R_{2}$ are $M_{2}, H_{1}$ and $G_{1}$, and of $R_{3}$ are $M_{3}, G_{2}$ and $K_{2}$. The intersection of the plane $L^{+}\left(u_{1}\right)$ with $D_{L}$ is the interval $K_{1} H_{2}$ which is the common boundary of $R$ and $R_{1}$. Similarly, $L^{+}\left(u_{2}\right) \cap D_{L}=G_{1} H_{1}=R \cap R_{2}$ and $L^{+}\left(u_{3}\right) \cap D_{L}=G_{2} K_{2}=R \cap R_{3}$. Note that $R_{i}\left[h_{i}\right]=L_{P}\left[h_{i}\right] \cap K(w), i=1,2,3$.

The sides of $D_{L}$ are identified as follows: $E: F_{1} M_{1} \rightarrow F_{2} M_{3} ; E_{1}: F_{2} M_{2} \rightarrow$ $F_{3} M_{1} ; E_{2}: F_{1} M_{2} \rightarrow F_{3} M_{3}$. Hence $D_{L}$ is a fundamental domain of $\left\langle E, E_{1}\right\rangle$, and therefore we have given a new proof that $\mathbf{Z}_{F}^{\times} /\{ \pm 1\}=\langle\epsilon, \epsilon+2\rangle$.

The set of convergents of $L_{P}$ is $\left\{g u, g \in \Gamma_{L}, u \in V_{L}^{\prime}\right\}$, where $V_{L}^{\prime}=\left\{w, u_{1}, u_{2}, u_{3}\right\}$.

Remark. Six sides of $R$ are identified as follows: $E: F_{1} K_{1} \rightarrow F_{2} K_{2} ; E_{1}: F_{2} H_{1} \rightarrow$ $F_{3} H_{2} ; E_{2}: F_{1} G_{1} \rightarrow F_{3} G_{2}$.

Example 5. Assume that $t>3$ is a positive integer which is not divisible by 3. Then $4 t^{2}+9$ is a square-free integer. Let $f(x)=x^{4}+t x^{3}-x^{2}-t x+1=$ $x\left(x^{2}-1\right)(x+t)+1$. Let $f(\epsilon)=0$. The discriminant of $f(x)$ is $\left(4 t^{2}+9\right)\left(t^{2}-4\right)^{2}$. Let $\eta=\epsilon-\epsilon^{-1}$. Since

$$
x^{-2} f(x)=\left(x-x^{-1}\right)^{2}+t\left(x-x^{-1}\right)+1,
$$

it follows that $\eta^{2}+t \eta+1=0, \eta=\left(-t \pm \sqrt{t^{2}-4}\right) / 2$, and $K=\mathbf{Q}(\eta)$ is a quadratic subfield of the totally real quartic field $F=\mathbf{Q}(\epsilon)$. We have $\epsilon^{2}-\eta \epsilon-1=0$, and the roots of $f(x)$ are

$$
\epsilon_{i, i+2}=\frac{\eta_{i}}{2} \pm \sqrt{\frac{\eta_{i}^{2}}{4}+1}, \quad \eta_{i}=-\frac{t}{2} \pm \sqrt{\frac{t^{2}}{4}-1}, \quad i=1,2 .
$$


Clearly, $\epsilon_{1} \epsilon_{3}=\epsilon_{2} \epsilon_{4}=-1$. Hence, $F_{i}=K\left(\sqrt{\eta_{i}^{2}+4}\right)$ and the discriminant of $F_{i}$ is

$$
D_{F}=D_{K}^{2} N\left(D_{F / K}\right)=\left(t^{2}-4\right)^{2} N\left(\eta_{i}^{2}+4\right)=\left(t^{2}-4\right)^{2}\left(4 t^{2}+9\right)
$$

provided $t^{2}-4$ is the discriminant of $K$, in which case $\mathbf{Z}_{K}^{\times} /\{ \pm 1\}=\left\langle\eta_{i}\right\rangle$, where $\mathbf{Z}_{K}^{\times}$is the unit group in the maximal order $\mathbf{Z}_{K}$ of $K$. Since $N\left(D_{F / K}\right)=4 t^{2}+9$ is square-free, $\{1, \epsilon\}$ is a basis of $\mathbf{Z}_{F / K}$ (see e.g. [7], p. 79). It follows that $\{1, \epsilon, \epsilon \eta, \eta\}$ or $\left\{1, \epsilon, \epsilon^{2}, \epsilon^{3}\right\}$ is a basis of $\mathbf{Z}_{F}$. The regulator obtained from the units $\epsilon_{1}, \epsilon_{2}$, and $\eta$ is of order $\log ^{3} t$ as $t \rightarrow \infty$. To show that the Galois group of $\mathbf{Q}(\epsilon)$ is $D_{4}$, it is enough to show that $F_{i}$ is not a normal field. But, if it is, then $\sqrt{\eta_{1}^{2}+4} \sqrt{\eta_{2}^{2}+4}=$ $\sqrt{N\left(\eta_{i}^{2}+4\right)}=\sqrt{4 t^{2}+9}$ belongs $F_{i}$ and therefore $D_{F}$ is divisible by $\left(4 t^{2}+9\right)^{2}$, which is not the case since $t>3$.

Let $E^{T}$ be the companion matrix of $f(x), E_{1}=E+I, E_{2}=E-E^{-1}$, and $E_{3}=E-I=E E_{2} E_{1}^{-1}$. Let $L_{P}$ be the axis of $E$. Since $\mathbf{Z}_{F}$ has a power basis, Lemma 9 is applicable. Now we shall show that the following 28 points are the vertices of $R=L_{P} \cap K(w)$.

The points $A=[1,1 / 2,1,-2 / t]$ and $B=[1,-1 / 2,1,-1 / 2-2 / t]$ with $\operatorname{det}(B)=$ $\operatorname{det}(A) \sim \frac{3}{8} t$ as $t \rightarrow \infty$ are the intersections of $L_{P}$ with $L^{+}(E), L^{+}\left(E_{2}\right), L^{+}\left(E_{3}\right)$ and with $L^{+}\left(E_{1}\right), L^{+}(E), L^{+}\left(E E_{3}\right)$ respectively. Denote $A_{1}=A[E], A_{2}=A\left[E_{2}\right]$, $A_{3}=A\left[E_{3}\right]$, and $B_{1}=B\left[E_{1}\right], B_{2}=B[E], B_{3}=B\left[E E_{2}\right]$.

The points $C=[1,-1 / 2,1,-t / 2+1 / t-1 / 4]$ and $D=[1,1 / 2,1,-t / 2+1 / t+1 / 4]$ with $\operatorname{det}(D)=\operatorname{det}(C) \sim \frac{7}{64} t^{2}$ as $t \rightarrow \infty$ are the intersections of $L_{P}$ with $L^{+}\left(E_{1}\right)$, $L^{+}\left(E_{1} E_{2}^{-1}\right), L^{+}(E)$ and with $L^{+}(E), L^{+}\left(E E_{1}^{-1}\right), L^{+}\left(E_{3}\right)$ respectively. Denote $C_{1}=C\left[E_{1}\right], C_{2}=C\left[E_{1} E_{2}^{-1}\right], C_{3}=C[E]$ and $D_{1}=D[E], D_{2}=D\left[E E_{1}^{-1}\right]$, $D_{3}=D\left[E_{3}\right]$.

Let $s=1 /\left(2 t^{2}-t-2\right)$. The points $K=[1,-s, 1,2 / t-t]$ and $L=[1, s, 1, s+2 / t-t]$ with $\operatorname{det}(L)=\operatorname{det}(K) \sim 3 / 4$ as $t \rightarrow \infty$ are the intersections of $L_{P}$ with $L^{+}(E)$, $L^{+}\left(E_{1} E_{2}^{-1}\right), L^{+}\left(E_{2}^{-1}\right)$ and $L^{+}\left(E_{1}\right), L^{+}\left(E_{1} E_{2}^{-1}\right), L^{+}\left(E_{1} E^{-1}\right)$ respectively. Denote $K_{1}=K[E], K_{2}=K\left[E_{1} E_{2}^{-1}\right], K_{3}=K\left[E_{2}^{-1}\right]$ and $L_{1}=L\left[E_{1}\right], L_{2}=L\left[E_{1} E_{2}^{-1}\right]$, $L_{3}=L\left[E_{1} E^{-1}\right]$.

The point $M=[1,0,1,-2 / t]$ with $\operatorname{det}(M)=\left(1-4 / t^{2}\right)^{2}$ is the intersection of $L_{P}$ with $L^{+}(E), L^{+}\left(E_{2}\right)$, and $L^{+}\left(E E_{2}\right)$. Denote $M_{1}=M[E], M_{2}=M\left[E_{2}\right], M_{3}=$ $M\left[E E_{2}\right]$. Note that the summit $q_{m}$ of the 3 -flat $L_{P}$ is $q_{m}=\frac{1}{4}\left(M+M_{1}+M_{2}+M_{3}\right)$ with $\operatorname{det}\left(q_{m}\right)=D_{F} / 4^{4}$.

To show that all the points enumerated above are extremal we shall use Minkowski reduction. (If for some $h \in \Gamma_{\infty}$ the point $X[h]$ is Minkowski reduced ([8], pp. 396-397), then $X$ is extremal). Below we shall indicate such an $h$ for one of the vertices in each of the $\Gamma_{L}$-orbits of vertices of $R$. The polytope $R$ is bounded by four octagons lying in $L^{+}\left(g^{ \pm 1}\right), g=E, E_{2}$, eight pentagons lying in $L^{+}\left(g^{ \pm 1}\right), g=E_{1}$, $E_{3}, E E_{1}^{-1}, E_{1} E_{2}^{-1}$, and four triangular faces lying in $L^{+}\left(g^{ \pm 1}\right), g=E E_{2}, E E_{2}^{-1}$. It has 28 vertices, 42 edges and 16 faces. The projections of the boundary of $R$ into a plane which is 'perpendicular' to its octagonal faces are shown in Figure 1.

Let

$$
U_{A}=\left[\begin{array}{llll}
1 & 1 & -t & -2 \\
0 & 1 & 0 & 1 \\
0 & 0 & t & 1 \\
0 & 0 & 1 & 0
\end{array}\right], \quad U_{B}=\left[\begin{array}{llll}
1 & 0 & -1 & 1-t \\
0 & 1 & 0 & 0 \\
0 & 0 & 1 & t \\
0 & 0 & 0 & 1
\end{array}\right]
$$



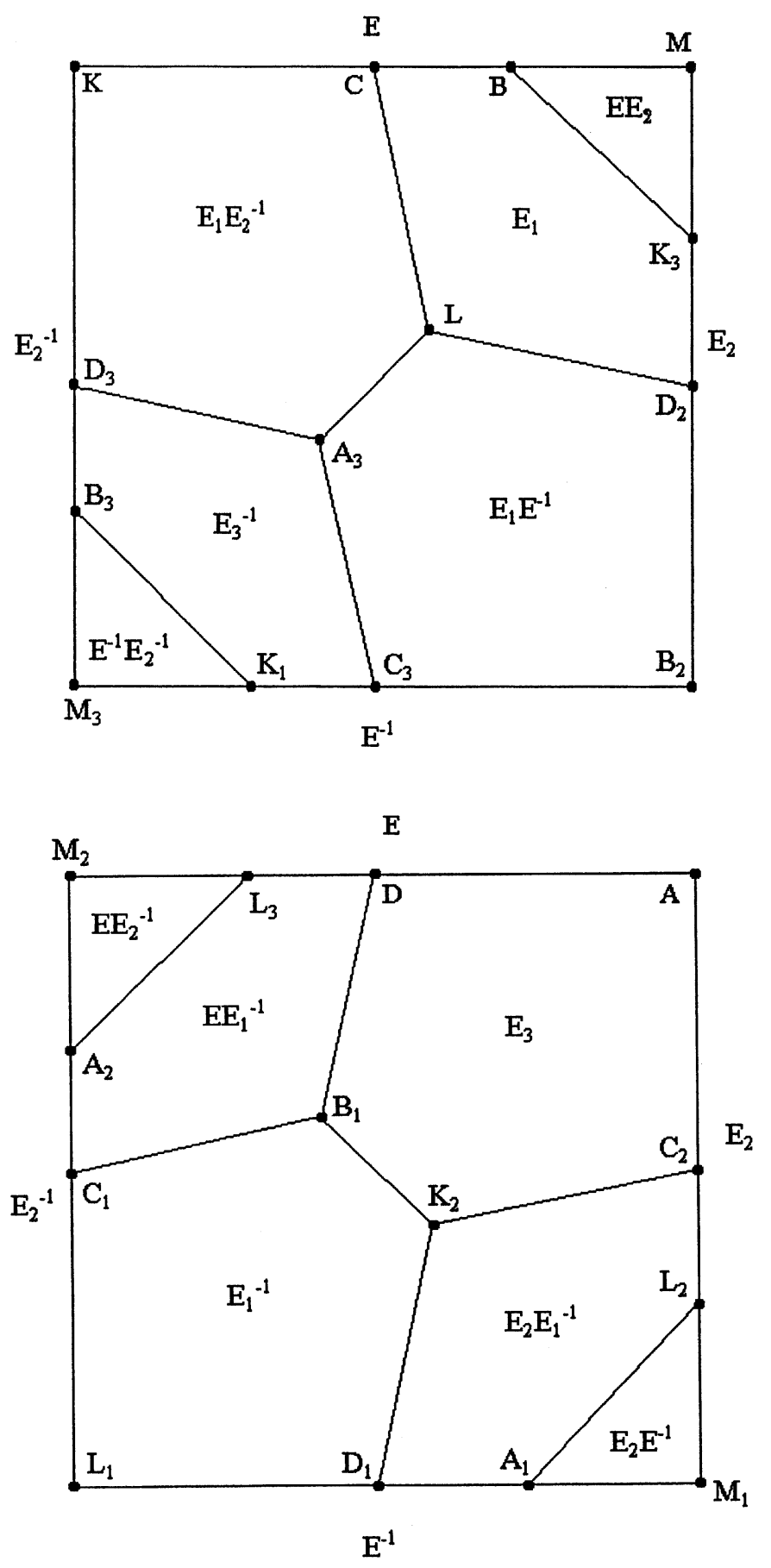

Figure 1. 
Let $b=1 / 2-2 / t, c=t / 2+1-4 / t, d=-1 / 2-2 / t$. Then

$$
A^{U}=\left[\begin{array}{llll}
1 & 1 / 2 & -2 / t & -1 / 2 \\
1 / 2 & 1 & 0 & -2 / t \\
-2 / t & 0 & 1 & -b \\
-1 / 2 & -2 / t & -b & c
\end{array}\right], B^{U}=\left[\begin{array}{llll}
1 & -1 / 2 & 0 & b \\
-1 / 2 & 1 & -2 / t & -1 / 2 \\
0 & -2 / t & 1 & 1 / 2 \\
b & -1 / 2 & 1 / 2 & c
\end{array}\right],
$$

where $A^{U}=A\left[U_{A}\right]$ and $B^{U}=B\left[U_{B}\right]$, are Minkowski reduced. Hence $A, A_{i}$ and $B$, $B_{i}, 1=1,2,3$, are extremal. Let

$$
U_{K}=\left[\begin{array}{llll}
1 & 0 & -1 & 0 \\
0 & 1 & t & 0 \\
0 & 0 & t+1 & -t \\
0 & 0 & 1 & -1
\end{array}\right], \quad U_{L}=\left[\begin{array}{llll}
1 & 0 & -1 & 1 \\
0 & 1 & t & -t \\
0 & 0 & 1 & t-1 \\
0 & 0 & 0 & 1
\end{array}\right]
$$

Let $a=2 t^{3}-t^{2}-2 t, b=3 t^{2}-t-4, c=-t^{3}+t^{2}+t, d=t-b$. Then

$$
a K^{U}=\left[\begin{array}{llll}
a & -t & -d & -2 a / t \\
-t & a & b & t^{2} \\
-d & b & a & c \\
-2 a / t & t^{2} & c & a
\end{array}\right], a L^{U}=\left[\begin{array}{llll}
a & t & t^{2} & b \\
t & a & 2 a / t & d \\
t^{2} & 2 a / t & a & c \\
b & d & c & a
\end{array}\right],
$$

where $K^{U}=K\left[U_{K}\right]$ and $L^{U}=L\left[U_{L}\right]$, are Minkowski reduced. Hence $K, K_{i}$ and $L, L_{i}, 1=1,2,3$, are extremal. For

$$
U=\left[\begin{array}{llll}
1 & 0 & -1 & -t \\
0 & 1 & 0 & 0 \\
0 & 0 & 1 & t \\
0 & 0 & 0 & 1
\end{array}\right]
$$

the matrix

$$
t M[U]=\left[\begin{array}{llll}
t & 0 & 0 & -2 \\
0 & t & -2 & 0 \\
0 & -2 & t & 0 \\
-2 & 0 & 0 & t
\end{array}\right]
$$

is Minkowski reduced. Thus, $M, M_{i}, i=1,2,3$, are extremal.

Reduction of $C$ and $D$ is more difficult. Let $t=22 k+l$. Let

$$
U_{D}=\left[\begin{array}{llll}
1 & 0 & 1 & u_{14} \\
0 & 1 & -t & u_{24} \\
0 & 0 & t-1 & u_{34} \\
0 & 0 & 1 & u_{44}
\end{array}\right]
$$

where $u_{44}=-[t / 11], u_{34}=u_{44}(t-1)+1, u_{14}=-9 k-a$, while

$$
u_{24}=44 k^{2}+(2 l+15) k+b,
$$

where $a=[l / 3]+1, b=[(2 l+1) / 3]$ if $1 \leq l \leq 10$, and

$$
u_{24}=44 k^{2}+(2 l-7) k+b,
$$

where $a=-[-l / 3], b=[(1-l) / 3]$ if $-11 \leq l \leq 0$. Then $D\left[U_{D}\right]$ is Minkowski reduced. For example, when $l=0$,

$$
D\left[U_{D}\right]=\left[\begin{array}{llll}
1 & 1 / 2 & 1 / 4+1 / t & 7 / 44+1 / t \\
1 / 2 & 1 & 1 / 4-1 / t & 1 / 11 \\
1 / 4+1 / t & 1 / 4-1 / t & 1 & 1 / 2 \\
7 / 44+1 / t & 1 / 11 & 1 / 2 & 7 / 44\left(t^{2}-4\right)
\end{array}\right] .
$$


Let

$$
U_{C}=\left[\begin{array}{llll}
1 & 0 & -1 & u_{14} \\
0 & 1 & t & u_{24} \\
0 & 0 & t+1 & u_{34} \\
0 & 0 & 1 & u_{44}
\end{array}\right]
$$

where $u_{44}=-[t / 11], u_{34}=u_{44}(t+1)+1, u_{14}=9 k+a$, while

$$
u_{24}=-44 k^{2}-(2 l+7) k-b,
$$

where $a=[l / 3], b=[(l+1) / 3]$ if $0 \leq l \leq 11$, and

$$
u_{24}=-44 k^{2}-(2 l-15) k-b,
$$

where $a=[l / 3]-1$, if $-10 \leq l \leq-1, l \neq-5$, and $a=[l / 3]-2$ if $l=-5$, and $b=[(1-2 l) / 3]$ if $-10 \leq l \leq-1$. Then $C\left[U_{C}\right]$ is Minkowski reduced. For example, when $l=0$,

$$
C\left[U_{C}\right]=\left[\begin{array}{llll}
1 & -1 / 2 & 1 / t-1 / 4 & -1 / 11 \\
-1 / 2 & 1 & 1 / t+1 / 4 & 7 / 44+1 / t \\
1 / t-1 / 4 & 1 / t+1 / 4 & 1 & 1 / 2 \\
-1 / 11 & 7 / 44+1 / t & 1 / 2 & 7 / 44\left(t^{2}-4\right)
\end{array}\right] .
$$

Hence, $C, C_{i}$ and $D, D_{i}, 1=1,2,3$, are extremal.

Thus, $R=L_{P} \cap K(w)$ is a fundamental domain of $\Gamma_{L}=\left\langle E, E_{1}, E_{2}\right\rangle$, and therefore $\mathbf{Z}_{F}^{\times} /\{ \pm 1\}=\langle\epsilon, \epsilon+1, \epsilon-1 / \epsilon\rangle=\langle\epsilon, \epsilon+1, \epsilon-1\rangle$.

Example 6. Let $f(x)=x^{4}+t x^{3}-x^{2}-t x-1=x\left(x^{2}-1\right)(x+t)-1$. Let $f(\epsilon)=0$. The field $F=\mathbf{Q}(\epsilon)$ is totally real and $\operatorname{Gal}(F)=S_{4}$. Assume that the discriminant of $f(x)$ is square-free. Then $\mathbf{Z}_{F}$ has a power basis, and Lemma 9 is applicable.

Let $E^{T}$ be the companion matrix of $f(x), E_{1}=E-I, E_{2}=E-E^{-1}$, and $E_{3}=E+I=E E_{2} E_{1}^{-1}$. Let $L_{P}$ be the axis of $E$. We shall show that the region $R=L_{P} \cap K(w)$ is the same as in Example 5 .

The point $A=[1,-1 / 2,1,-1-2 / t]$ with $\operatorname{det}(A) \sim \frac{3}{8} t$ as $t \rightarrow \infty$ is the intersection of $L_{P}$ with $L^{+}(E), L^{+}\left(E_{2}\right)$ and $L^{+}\left(E_{3}\right)$. Denote $A_{1}=A[E], A_{2}=A\left[E_{2}\right], A_{3}=$ $A\left[E_{3}\right]$. Let $c=-1 / 2+2 / t$,

$$
U_{A}=\left[\begin{array}{llll}
1 & 0 & -1 & -t \\
0 & 1 & 0 & -2 \\
0 & 0 & 1 & t \\
0 & 0 & 0 & 1
\end{array}\right], \quad A^{U}=\left[\begin{array}{llll}
1 & -1 / 2 & -2 / t & c \\
-1 / 2 & 1 & 0 & -c \\
-2 / t & 0 & 1 & c+2 / t \\
c & -c & c+2 / t & t / 2-6 c
\end{array}\right] .
$$

Then $A\left[U_{A}\right]=A^{U}$ is Minkowski reduced. Hence $A, A_{1}, A_{2}$ and $A_{3}$ are extremal.

The point $B_{3}=[1,1 / 2,1,1 / 2]$ with $\operatorname{det}(B)=(6 t-19) / 16$ is the intersection of $L_{P}$ with $L^{+}\left(E^{-1} E_{2}^{-1}\right), L^{+}\left(E_{2}^{-1}\right)$ and $L^{+}\left(E_{3}^{-1}\right)$. Denote $B=B_{3}\left[E^{-1} E_{2}^{-1}\right], B_{1}=$ $B_{3}\left[E_{3}^{-1}\right], B_{2}=B_{3}\left[E_{2}^{-1}\right]$. Since $B_{3}$ is an integral matrix, it is extremal.

The points $C=[1,1 / 2,1,-t / 2+7 / 4+1 /(2 t-4)]$ and $D=[1,-1 / 2,1,-t / 2-7 / 4+$ $1 /(2 t+4)]$ with $\operatorname{det}(C), \operatorname{det}(D) \sim \frac{7}{64} t^{2}$ as $t \rightarrow \infty$ are the intersections of $L_{P}$ with $L^{+}(E), L^{+}\left(E_{1}\right), L^{+}\left(E_{1} E_{2}^{-1}\right)$ and with $L^{+}(E), L^{+}\left(E_{3}\right), L^{+}\left(E E_{1}^{-1}\right)$ respectively. Denote $C_{1}=C\left[E_{1}\right], C_{2}=C\left[E_{1} E_{2}^{-1}\right], C_{3}=C[E]$ and $D_{1}=D[E], D_{2}=D\left[E E_{1}^{-1}\right]$, $D_{3}=D\left[E_{3}\right]$. As in Example 5, reduction of $C$ and $D$ is divided into particular cases modulo 22 . We consider only one case of such reduction. Let $t=22 k+13$. 
Let

$$
U_{C}=\left[\begin{array}{llll}
1 & 0 & -1 & -u_{14} \\
0 & 1 & -t & -u_{24} \\
0 & 0 & t-1 & u_{34} \\
0 & 0 & 1 & u_{44}
\end{array}\right]
$$

where $u_{44}=2+2 k, u_{34}=u_{44}(t-1)+1, u_{14}=9 k+6, u_{24}=44 k^{2}+55 k+19$. Then

$$
C\left[U_{C}\right]=\left[\begin{array}{llll}
1 & 1 / 2 & -1 / 4+c & 1 / 22+c \\
1 / 2 & 1 & -1 / 4-c & -1 / 4-1 / 22 \\
1 / 4+c & 1 / 4-c & 1 & c \\
1 / 22+c & -1 / 4-1 / 22 & c & 7 / 44 t^{2}+3 / 11 t-31 / 22-c
\end{array}\right],
$$

where $c=1 /(22+44 s)$, is Minkowski reduced. Hence $C$ is extremal. Similarly, the other cases of reduction of $C$ and $D$ can be considered.

Denote by $K_{3}$ the intersection of $L_{P}$ with $L^{+}\left(E_{3}\right), L^{+}\left(E_{2}\right)$ and $L^{+}\left(E E_{2}\right)$, and by $L_{3}$ the intersection of $L_{P}$ with $L^{+}(E), L^{+}\left(E E_{2}^{-1}\right)$ and $L^{+}\left(E E_{1}^{-1}\right)$. Let $K=K_{3}\left[E_{2}\right]$, $K_{1}=K[E], K_{2}=K\left[E_{1} E_{2}^{-1}\right]$ and $L=L_{3}\left[E E_{1}^{-1}\right], L_{1}=L\left[E_{1}\right], L_{2}=L\left[E_{1} E_{2}^{-1}\right]$. Let

$$
U_{K}=\left[\begin{array}{llll}
1 & 0 & -1 & 0 \\
0 & 1 & -t & 0 \\
0 & 0 & t-1 & t \\
0 & 0 & 1 & 1
\end{array}\right], \quad U_{L}=\left[\begin{array}{llll}
1 & 0 & 1 & 1 \\
0 & 1 & t & t \\
0 & 0 & 1 & t+1 \\
0 & 0 & 0 & 1
\end{array}\right]
$$

and $a_{1}=t(t-2)(2 t+3), b_{1}=(t-4) / a_{1}, c_{1}=\left(3 t^{2}-4\right) / a_{1}, d_{1}=\left(t^{2}-3 t-4\right) / a_{2}$, $a_{2}=t(t+2)(2 t-5), b_{2}=(t+4) / a_{2}, c_{2}=\left(3 t^{2}-3 t-8\right) / a_{2}, d_{2}=-\left(t^{2}+2 t-8\right) / a_{2}$. Then

$$
\begin{aligned}
K\left[U_{K}\right]= & {\left[\begin{array}{llll}
1 & -2 / t-b_{1} & c_{1} & 2 / t-2 b_{1} \\
-2 / t-b_{1} & 1 & d_{1} & t b_{1} \\
c_{1} & d_{1} & 1 & 1 / 2-t b_{1} / 2 \\
2 / t-2 b_{1} & t b_{1} & 1 / 2-t b_{1} / 2 & 1
\end{array}\right], } \\
L_{3}\left[U_{L}\right]= & {\left[\begin{array}{llll}
1 & -2 / t-b_{2} & -t b_{2} & c_{2} \\
-2 / t-b_{2} & 1 & 2 b_{2} & d_{2} \\
-t b_{2} & 2 b_{2} & 1 & 1 / 2-t b_{2} / 2 \\
c_{2} & d_{2} & 1 / 2-t b_{2} / 2 & 1
\end{array}\right] }
\end{aligned}
$$

are Minkowski reduced. Hence $K$ and $L$ are extremal. Note that $\operatorname{det}(K) \sim 3 / 4$, $\operatorname{det}(L) \sim 3 / 4$ as $t \rightarrow \infty$.

The point $M=[1,2 / t, 1,2 / t]$ with $\operatorname{det}(M)=\left(1-4 / t^{2}\right)^{2}-4 / t^{2}$ is the intersection of $L_{P}$ with $L^{+}(E), L^{+}\left(E_{2}\right)$ and $L^{+}\left(E E_{2}\right)$. Denote $M_{1}=M[E], M_{2}=M\left[E_{2}\right]$, $M_{3}=M\left[E E_{2}\right]$. Let

$$
U_{M}=\left[\begin{array}{llll}
1 & 0 & -1 & -t \\
0 & 1 & 0 & -2 \\
0 & 0 & 1 & t \\
0 & 0 & 0 & 1
\end{array}\right], \quad M_{U}=\left[\begin{array}{llll}
1 & 2 / t & 0 & -2 / t \\
2 / t & 1 & 0 & 0 \\
0 & 0 & 1 & 2 / t \\
-2 / t & 0 & 2 / t & 1
\end{array}\right]
$$

Since $M\left[U_{M}\right]=M_{U}$ is Minkowski reduced, the points $M, M_{1}, M_{2}, M_{3}$ are extremal.

Thus, the polytope $R=L_{P} \cap K(w)$ is the same as in Example 5 (see Figure 1). It is a fundamental domain of $\Gamma_{L}=\left\langle E, E_{1}, E_{2}\right\rangle$. Hence $\mathbf{Z}_{F}^{\times} /\{ \pm 1\}=\langle\epsilon, \epsilon+1, \epsilon-1 / \epsilon\rangle=$ $\langle\epsilon, \epsilon+1, \epsilon-1\rangle$. 


\section{UNits IN FIELDS WITH SIGNATURE $(2,1)$}

Let $n=4$. Let $g \in \Gamma$. Assume that the characteristic polynomial of $g$ is irreducible with signature $(2,1)$. Let $\epsilon_{1}, \epsilon_{2}$ be real and $\epsilon_{3}, \epsilon_{4}=\bar{\epsilon}_{3}$ non-real complex eigenvalues of $g$. Let $g a_{i}=\epsilon_{i} a_{i}$. Assume that the field $F=\mathbf{Q}\left(\epsilon_{1}\right)$ is dihedral and $K$ is its real quadratic subfield. Let $\sigma$ be the non-trivial automorphism of $F / K$. Then $\sigma\left(\epsilon_{i}\right)=\epsilon_{i+1}, i=1,3$. Hence $\sigma\left(A_{1}\right)=A_{2}$ and $\sigma\left(A_{3}\right)=A_{3}$, where $A_{1}, A_{2}$, and $A_{3}$ are vertices of $L_{P}$, the axis of $g$. Thus, the entries of $A_{1}+A_{2}$ and of $A_{3}$ lie in $K$, and $q_{m}=\left(A_{1}+A_{2}+2 A_{3}\right) / 3$ is rational over $K$. Hence $L_{P}$ and the vertices of $R=L_{P} \cap K(w)$ are rational over $K$. It follows that $F_{L}=K$ in this case. In Examples 7 and 9 below, the signature of the field $F$ is $(2,1), F$ has a real quadratic subfield $K$, and $F_{L}=K$. In Example 8, the degree of $F_{L}$ is six.

Example 7. Let $f(x)=x^{4}+t x^{3}+x^{2}+t x+1=x\left(x^{2}+1\right)(x+t)+1$. Let $f(\epsilon)=0$, where $\epsilon \in \mathbf{R}$. The discriminant of $f(x)$ is $-\left(4 t^{2}-9\right)\left(t^{2}+4\right)^{2}$. Let $\eta=\epsilon+\epsilon^{-1}$. Since

$$
x^{-2} f(x)=\left(x+x^{-1}\right)^{2}+t\left(x+x^{-1}\right)-1,
$$

it follows that $\eta^{2}+t \eta-1=0, \eta=\left(-t \pm \sqrt{t^{2}+4}\right) / 2$, and $K=\mathbf{Q}(\sqrt{d}), d=t^{2}+4$, is a quadratic subfield of the dihedral quartic field $F=\mathbf{Q}(\epsilon)$ with signature $(2,1)$. We have $\psi(\epsilon)=\epsilon^{2}-\eta \epsilon+1=0$, and the roots of $f(x)$ are

$$
\epsilon_{i, i+2}=\frac{1}{2}\left(\eta_{i} \pm \sqrt{\eta_{i}^{2}-4}\right), \quad \eta_{i}=\frac{1}{2}\left(-t \mp \sqrt{t^{2}+4}\right), \quad i=1,2 .
$$

Hence $\epsilon_{1} \epsilon_{3}=\epsilon_{2} \epsilon_{4}=-1$. If $N\left(\eta^{2}-4\right)=4 t^{2}-9$, the norm of the discriminant of $\psi(x)$, is square-free, then $\{1, \epsilon\}$ is a basis of $\mathbf{Z}_{F / K}$. Hence $\{1, \epsilon, \eta \epsilon, \eta\}$ or $\left\{1, \epsilon, \epsilon^{2}, \epsilon^{3}\right\}$ is a basis of $\mathbf{Z}_{F}$ provided $\eta$ is a fundamental unit of $K$.

Let $E^{T}$ be the companion matrix of $f(x)$. Then $E^{T} a_{i}=\epsilon_{i} a_{i}$, where $a_{i}=$ $\left(1, \epsilon_{i}, \epsilon_{i}^{2}, \epsilon_{i}^{3}\right)$. Denote $E_{1}=E+E^{-1}$. Let $M$ be the intersection of $L_{P}, L^{+}(E)$, and $L^{+}\left(E_{1}\right)$. Then

$$
M[x]=\mu_{1}\left(x, a_{1}\right)^{2}+\mu_{3}\left(x, a_{3}\right)^{2}+\mu_{2}\left|\left(x, a_{2}\right)\right|^{2},
$$

where $\mu_{1,3}=\left(1+\left(t \pm\left(2 t \sqrt{d}+2 t^{2}-12\right)^{1 / 2}\right) / \sqrt{d}\right) / 4, \mu_{2}=(1-t / \sqrt{d}) / 2$, and $\operatorname{det}(M)=\left(4 t^{2}-9\right)\left(t^{2}+2-t \sqrt{d}\right) / 2$.

Let $b=-2 t(\eta \sqrt{d})^{-1}-3(2 \sqrt{d})^{-1}, c=-2 t(\eta \sqrt{d})^{-1}+3(2 \sqrt{d})^{-1}, e=5(4 \eta \sqrt{d})^{-1}$ and

$$
h=\left[\begin{array}{llll}
1 & 0 & 1 & t \\
0 & 1 & 0 & 0 \\
0 & 0 & 1 & t \\
0 & 0 & 0 & 1
\end{array}\right], \quad M_{0}=\left[\begin{array}{llll}
1 & b & e & 0 \\
b & 1 & 0 & -e \\
e & 0 & 1 & c \\
0 & -e & c & 1
\end{array}\right]
$$

Then $M_{0}=M[h]$ is Minkowski reduced (see e.g. 8], p. 397). Hence the points $M$, $M[E], M\left[E_{1}\right]$, and $M\left[E E_{1}\right]$ are extremal, and they are the vertices of the 'square' $R=L_{P} \cap K(w)$, which is a fundamental domain of $\left\langle E, E_{1}\right\rangle$. Thus $\mathbf{Z}_{F}^{\times} /\{ \pm 1\}=\langle\epsilon, \eta\rangle$.

Note that $q_{m}=\left(M+M[E]+M\left[E_{1}\right]+M\left[E E_{1}\right]\right) / 4$ is the summit of $L_{P}$.

Example 8 (cf. [20]). Let $t \geq 4$ and $f_{t}(x)=f(x)=x^{4}+t x^{3}+x^{2}+t x-1=$ $x\left(x^{2}+1\right)(x+t)-1$. Note that $f_{-t}(-x)=f_{t}(x)$. Let $\epsilon$ and $\epsilon_{1}$ be the real and $\epsilon_{2}, \overline{\epsilon_{2}}$ non-real complex roots of $f(x)=0$. The signature of $F=\mathbf{Q}(\epsilon)$ is $(2,1)$, and $\operatorname{Gal}(F)=S_{4}$. Assume that the discriminant of $F$ is square-free. Then $\left\{1, \epsilon, \epsilon^{2}, \epsilon^{3}\right\}$ is a basis of the maximal order $\mathbf{Z}_{F}$ of $F$. 
Let $E^{T}$ be the companion matrix of $f(x)$. Let $E_{1}=E^{2}(E+t I)$. Let $L_{P}$ be the axis of $E$. It can be identified with the set

$$
F\left(\nu_{1}, \nu_{2}, \nu_{3}\right)=\nu_{1} a^{T} a+\nu_{2} a_{1}^{T} a_{1}+\nu_{3}\left(a_{2 R}^{T} a_{2 R}+a_{2 I}^{T} a_{2 I}\right),
$$

where $\nu_{k}>0, \nu_{1}+\nu_{2}+\nu_{3}=1$. Here $a=\left(1, \epsilon, \epsilon^{2}, \epsilon^{3}\right), a_{i}=\left(1, \epsilon_{i}, \epsilon_{i}^{2}, \epsilon_{i}^{3}\right), i=1,2$, are the eigenvectors of $E$ corresponding to its eigenvalues $\epsilon, \epsilon_{1}, \epsilon_{2}$ respectively. Let $A$ and $B$ be the intersections of $L_{P}$ with $L^{+}(E), L^{+}\left(E_{1}\right)$ and with $L^{+}\left(E^{-1}\right), L^{+}\left(E_{1}^{-1}\right)$ respectively.

Let $\alpha=\left|\epsilon_{2}\right|^{2}=-1 /\left(\epsilon \epsilon_{1}\right)$. Then $\alpha \rightarrow 1$ as $t \rightarrow \infty$. Over $F_{L}=\mathbf{Q}(\alpha)$ we have $f(x)=f_{1}(x) f_{2}(x)$, where

$$
\begin{aligned}
& f_{1}(x)=x^{2}+t \frac{\alpha+1}{\alpha^{2}+1} x-\frac{1}{\alpha}, \\
& f_{2}(x)=x^{2}+t \frac{\alpha^{2}-\alpha}{\alpha^{2}+1} x+\alpha,
\end{aligned}
$$

so that $f_{i}\left(\epsilon_{i}\right)=0, i=1,2$. The degree of $F_{L}$ is 6 , since $g(\alpha)=0$, where $g(x)=$ $x^{6}-x^{5}+\left(t^{2}+1\right) x^{4}-2 x^{3}-\left(t^{2}+1\right) x-x-1$.

Let $d_{1}$ be the discriminant of $f_{1}(x)$. Let $\nu_{1,2}=\mu_{1} \pm \mu_{2} \sqrt{d_{1}}, \mu_{3}=\nu_{3}, a^{T} a=$ $M_{1}+M_{2} \sqrt{d_{1}}, a_{1}^{T} a_{1}=M_{1}-M_{2} \sqrt{d_{1}}$. Then $L_{P}$ can be identified with the set $\mu_{1} M_{1}+$ $\mu_{2} d_{1} M_{2}+\mu_{3} M_{3}$, where the entries of $M_{k}, k=1,2,3$, belong to $F_{L}$. Thus, the summit $q_{m}$ of $L_{P}, L_{P}$ itself and all the vertices of $R=L_{P} \cap K(w)$ are rational over $F_{L}$.

Let $\Delta_{A}$ be the determinant of the system of three linear equations

$$
\begin{aligned}
2 \mu_{1}+\mu_{3} & =1 \\
\left(\mu_{1} M_{1}+\mu_{2} d_{1} M_{2}+\mu_{3} M_{3}\right)[w E] & =1 \\
\left(\mu_{1} M_{1}+\mu_{2} d_{1} M_{2}+\mu_{3} M_{3}\right)\left[w E_{1}\right] & =1
\end{aligned}
$$

with respect to $\mu_{1}, \mu_{2}, \mu_{3}$. Then

$$
\begin{aligned}
D_{A} & =\Delta_{A}\left(\alpha^{2}+1\right) \alpha /(4 t) \\
& =\alpha^{5}+2 \alpha^{4}+\left(2 t^{2}-1\right) \alpha^{3}+\left(t^{2}-2\right)\left(\alpha^{2}-\alpha\right)-2 .
\end{aligned}
$$

Let $\Delta_{B}$ be the determinant of the system of three similar linear equations where $E$ and $E_{1}$ are replaced by $E$ and $E E_{1}^{-1}$. Then

$$
\begin{aligned}
D_{B} & =\Delta_{B}\left(\alpha^{2}+1\right) /(4 t) \\
& =-\left(\alpha^{3}+\left(t^{2}+5\right) \alpha+t^{2}+2\right) .
\end{aligned}
$$

Let

$$
U_{A}=\left[\begin{array}{llll}
1 & 0 & 0 & -1 \\
0 & 1 & 0 & t \\
0 & 0 & t & 1 \\
0 & 0 & 1 & 0
\end{array}\right], \quad U_{B}=\left[\begin{array}{llll}
1 & 0 & 1 & t \\
0 & 1 & 0 & 2 \\
0 & 0 & 1 & t \\
0 & 0 & 0 & 1
\end{array}\right]
$$


and let $A_{U}=A\left[U_{A}\right]=\left(a_{i j}\right)$ and $B_{U}=B\left[U_{B}\right]=\left(b_{i j}\right)$. Then $a_{11}=a_{22}=a_{33}=1$,

$$
\begin{aligned}
2 t D_{A} a_{12}= & (1-\alpha)\left(1+\alpha^{2}-\alpha^{3}\right) t^{2}-4 \alpha\left(2-\alpha+2 \alpha^{2}-\alpha^{3}\right) \\
2 t D_{A} a_{13}= & \left(\alpha-\alpha^{3}\right) t^{4}+\left(1+5 \alpha-7 \alpha^{2}+4 \alpha^{3}+2 \alpha^{4}-\alpha^{5}\right) t^{2}-6-14 \alpha^{2} \\
& +6 \alpha^{3}-8 \alpha^{4}+6 \alpha^{5} \\
2 D_{A} a_{14}= & (1-\alpha)\left(1+\alpha-4 \alpha^{2}\right) t^{2}-6-3 \alpha-13 \alpha^{2}+2 \alpha^{3}-7 \alpha^{4}+5 \alpha^{5} \\
2 D_{A} a_{23}= & (1-\alpha)\left(\alpha+6 \alpha^{2}+\alpha^{3}\right) t^{2}+11-3 \alpha+18 \alpha^{2}-9 \alpha^{3}+7 \alpha^{4}-6 \alpha^{5}, \\
2 t D_{A} a_{24}= & \left(1+2 \alpha-2 \alpha^{2}+7 \alpha^{3}+\alpha^{4}-\alpha^{5}\right) t^{2}-6+8 \alpha-18 \alpha^{2}+14 \alpha^{3} \\
& -12 \alpha^{4}+6 \alpha^{5} \\
2 t D_{A} a_{34}= & 4\left(\alpha^{2}-\alpha^{3}\right) t^{4}+\left(9-9 \alpha+23 \alpha^{2}-14 \alpha^{3}+5 \alpha^{4}-4 \alpha^{5}\right) t^{2} \\
& +12-8 \alpha+32 \alpha^{2}-20 \alpha^{3}+20 \alpha^{4}-12 \alpha^{5} \\
D_{A} a_{44}= & \left(2 \alpha+3 \alpha^{2}-3 \alpha^{3}\right) t^{2}+8+12 \alpha^{2}+\alpha^{3}+2 \alpha^{4}-3 \alpha^{5}
\end{aligned}
$$

and $b_{11}=b_{22}=b_{33}=1$,

$$
\begin{aligned}
2 t D_{B} b_{12}= & 4\left(\alpha^{3}-\alpha\right) t^{4}+\left(-3-9 \alpha-12 \alpha^{2}+10 \alpha^{3}-4 \alpha^{4}+4 \alpha^{5}\right) t^{2} \\
& -4+2 \alpha-14 \alpha^{2}+8 \alpha^{3}-10 \alpha^{4}+6 \alpha^{5}, \\
2 D_{B} b_{13}= & \left(\alpha^{2}-1\right)(2-3 \alpha) t^{2}-4-11 \alpha+11 \alpha^{2}-4 \alpha^{3}+5 \alpha^{4}-3 \alpha^{5}, \\
2 t D_{B} b_{14}= & \left(\alpha^{2}-1\right)(\alpha+2) t^{4}+\left(-7-12 \alpha-2 \alpha^{2}-\alpha^{3}+\alpha^{4}+\alpha^{5}\right) t^{2} \\
& +4 \alpha\left(1-\alpha+\alpha^{2}-\alpha^{3}\right), \\
2 t D_{B} b_{23}= & 2\left(\alpha^{3}-\alpha\right) t^{4}+\left(5+4 \alpha-7 \alpha^{2}-2 \alpha^{3}-2 \alpha^{4}+2 \alpha^{5}\right) t^{2} \\
& +8+4 \alpha+8 \alpha^{2}-4 \alpha^{5}, \\
2 D_{B} b_{24}= & 2\left(\alpha^{3}-\alpha\right) t^{4}+\left(1-8 \alpha-7 \alpha^{2}+6 \alpha^{3}-2 \alpha^{4}+2 \alpha^{5}\right) t^{2} \\
& -3-13 \alpha-8 \alpha^{2}+5 \alpha^{3}-7 \alpha^{4}+4 \alpha^{5}, \\
2 t D_{B} b_{34}= & 2\left(\alpha-\alpha^{3}\right) t^{4}+\left(7+7 \alpha-4 \alpha^{2}-10 \alpha^{3}+2 \alpha^{4}-2 \alpha^{5}\right) t^{2} \\
& +10+14 \alpha+8 \alpha^{2}+6 \alpha^{3}-2 \alpha^{4}-8 \alpha^{5}, \\
D_{B} b_{44}= & \left(2-3 \alpha-5 \alpha^{2}\right) t^{2}-4-5 \alpha-4 \alpha^{2}+7 \alpha^{3}-6 \alpha^{4} .
\end{aligned}
$$

Let $c=\left(\alpha^{2}+1\right)^{2}$. The identity

$$
t^{2}\left(\alpha^{3}-\alpha\right)=c-\left(\frac{c}{\alpha t}\right)^{2}+\frac{c^{3}}{\alpha^{2} t^{2}\left(\alpha^{2} t^{2}+c\right)}
$$

implies $a_{i j} \rightarrow 0$ and $b_{i j} \rightarrow 0$ as $t \rightarrow \infty$ for $i \neq j$. It follows that $\operatorname{det}(A) \sim 1$ and $\operatorname{det}(B) \sim 3$ as $t \rightarrow \infty$. Thus, $A_{U}$ and $B_{U}$ are Minkowski reduced. Hence the points $A, A[E], A\left[E_{1}\right], B, B\left[E^{-1}\right]$, and $B\left[E_{1}^{-1}\right]$ are extremal, and they are the vertices of the hexagon $R=L_{P} \cap K(w)$. Thus, $R$ is a fundamental domain of $\left\langle E, E_{1}\right\rangle$, and $\mathbf{Z}_{F}^{\times} /\{ \pm 1\}=\langle\epsilon, \epsilon+t\rangle$.

Example 9. Let

$f(x)=x^{4}+s t x^{3}+\left(t-\alpha s^{2}\right) x^{2}+s\left(t^{2}+2 \alpha\right) x-\alpha=x\left(x^{2}+t\right)(x+s t)-\alpha(s x-1)^{2}$,

where $t \geq s$ are positive integers and $\alpha= \pm 1$. Let $d=t^{2}+4 \alpha$. It can be easily verified that

$$
f(x)=\left(x^{2}+s \eta x-\alpha / \eta\right)\left(x^{2}-\alpha s x / \eta+\eta\right),
$$


where $\eta=\frac{1}{2}(t+\sqrt{d})$ satisfies the equation $\eta^{2}-t \eta-\alpha=0$. The roots of $f(x)=0$ are

$$
\epsilon_{i, i+2}=\frac{1}{2}\left(-s \eta_{i} \pm \sqrt{s^{2} \eta_{i}^{2}+4 \alpha / \eta_{i}}\right), \quad \eta_{i}=\frac{1}{2}(t \pm \sqrt{d}), \quad i=1,2
$$

where $\eta_{1}=\eta$ and real $\epsilon_{1}$ and $\epsilon_{3}$ are the roots of $x^{2}+s \eta x-\alpha / \eta=0$. The discriminant of $f(x)$ is $\Delta(f)=-d^{2}\left(4 s^{2} t^{3}+12 \alpha s^{2} t-s^{4}+16 \alpha\right)\left(1+s^{2} t\right)^{2}$. Let $\epsilon=\epsilon_{1}$. Since $\eta=\left(\epsilon^{2}+t\right) /(1-s \epsilon), K=\mathbf{Q}(\eta)$ is a quadratic subfield of the dihedral quartic field $F=\mathbf{Q}(\epsilon)$ with signature $(2,1)$. Assume that $\eta$ is a fundamental unit of $K$. Then $\{1, \eta\}$ is a basis of $\mathbf{Z}_{K}$. Let $c=t s^{2}+1$. Denote $p(x)=\left(s+\left(\alpha t-s^{2}\right) x+\alpha x^{3}\right) / c$. Then $\epsilon \eta=p(\epsilon)$. Assume that $4 s^{2} t^{3}+12 \alpha s^{2} t-s^{4}+16 \alpha$ is square-free. Then $\left\{1, \epsilon, \epsilon^{2}, p(\epsilon)\right\}$ is a basis of $\mathbf{Z}_{F}$, and the discriminant of $F$ is $D_{F}=\Delta(f) /\left(t s^{2}+1\right)^{2}$.

Let $a_{i}=\left(1, \epsilon_{i}, \epsilon_{i}^{2}, p\left(\epsilon_{i}\right)\right)$. Let $E_{0}$ be the companion matrix of $f$. Denote

$$
\tau=\left[\begin{array}{llll}
1 & 0 & 0 & 0 \\
0 & 1 & 0 & 0 \\
0 & 0 & 1 & 0 \\
s / c & \left(\alpha t-s^{2}\right) / c & 0 & \alpha / c
\end{array}\right] .
$$

Let $E^{T}=\tau E_{0} \tau^{-1}, E_{1}=p(E), E_{2}=E_{1} E^{-1}$. Then $E^{T} a_{i}=\epsilon_{i} a_{i}$, and the axis $L_{P}$ of $E$ has equation $q[x]=\mu_{1}\left(x, a_{1}\right)^{2}+\mu_{3}\left(x, a_{3}\right)^{2}+\mu_{2}\left|\left(x, a_{2}\right)\right|^{2}, \mu_{i}>0, \mu_{1}+\mu_{3}+\mu_{2}=1$.

First let $\alpha=1$. Let $A$ and $B$ be the intersections of $L_{P}$ with $L^{+}(E), L^{+}\left(E_{1}\right)$ and with $L^{+}(E), L^{+}\left(E_{2}^{-1}\right)$ respectively. Then $\operatorname{det}(A)=\operatorname{det}(B)$. Let $a_{i i}=b_{i i}=1$, $i=1,2,3, a_{44}=b_{44}=\sqrt{d}-1+\eta^{-2}, a_{23}=b_{13}=0, a_{14}=b_{24}=\eta^{-1}$,

$$
\begin{aligned}
& a_{24}=-b_{14}=\left(-\frac{s}{2 \sqrt{d}}+\frac{t}{s \eta^{2} \sqrt{d}}\right)\left(1-\eta^{-1}\right), \\
& a_{34}=-b_{34}=\left(\frac{s}{2 \eta \sqrt{d}}+\frac{t}{s \eta \sqrt{d}}\right)\left(1-\eta^{-1}\right), \\
& a_{13}=-b_{23}=\frac{1}{s \eta}-\frac{2}{s \eta \sqrt{d}}-\frac{s}{2 \eta^{2} \sqrt{d}} \\
& a_{12}=-b_{12}=\frac{1}{s \eta^{2}}-\frac{2}{s \eta^{2} \sqrt{d}}+\frac{s}{2 \eta \sqrt{d}} .
\end{aligned}
$$

Let

$$
U_{A}=\left[\begin{array}{cccc}
1 & 0 & 0 & 1 \\
0 & 1 & 0 & 0 \\
0 & 0 & 1 & s \\
0 & 0 & 0 & 1
\end{array}\right], \quad U_{B}=\left[\begin{array}{cccc}
1 & 0 & t & 0 \\
0 & 1 & 0 & 1 \\
0 & 0 & 1 & 0 \\
0 & 1 & s & 1
\end{array}\right]
$$

Then $A_{U}=\left(a_{i j}\right)=A\left[U_{A}\right]$ and $B_{U}=\left(b_{i j}\right)=B\left[E_{2}^{-1}\right]\left[U_{B}\right]$ are Minkowski reduced. Hence the points $A, A[E], A\left[E_{1}\right], B, B[E], B\left[E_{2}^{-1}\right]$ are extremal, and they are the vertices of the hexagon $R=L_{P} \cap K(w)$.

Now let $\alpha=-1$. Let $A$ and $B$ be the intersections of $L_{P}$ with $L^{+}\left(E^{-1}\right)$, $L^{+}\left(E_{2}\right)$ and with $L^{+}\left(E_{1}\right), L^{+}\left(E_{2}\right)$ respectively. Then $\operatorname{det}(A)=\operatorname{det}(B)$. Note that if $A=q\left(\gamma_{1}, \gamma_{2}, \gamma_{3}\right)$, then $B=q\left(\gamma_{3}, \gamma_{2}, \gamma_{1}\right)$. Here $\gamma_{2}=\eta$ and $\sigma\left(\gamma_{1}\right)=\gamma_{3}$, where $\sigma \in \operatorname{Gal}(F / K)$. 
Let $a_{i i}=b_{i i}=1, i=1,2,3, a_{44}=b_{44}=\sqrt{d}-1-2 \eta^{-1}+\eta^{-2}-4 /(t \eta)$, $a_{14}=-b_{24}=-(1-2 / t) / \eta, a_{23}=b_{13}=-2 / t$,

$$
\begin{aligned}
& a_{12}=-b_{23}=-\frac{s}{2 t \eta}-\frac{1}{s \eta^{2}} \\
& a_{13}=-b_{12}=\frac{s}{2 \eta^{2} t}+\frac{1}{s \eta} \\
& a_{24}=b_{34}=\left(\frac{s}{2 \sqrt{d}}\left(1-\frac{2}{t}\right)-\frac{t-2}{s \eta^{2} \sqrt{d}}\right)\left(1+\eta^{-1}\right) \\
& a_{34}=b_{14}=\frac{2 t-s^{2}}{2 s \eta \sqrt{d}}\left(1+\eta^{-1}\right)(1-2 / t)
\end{aligned}
$$

Let

$$
U_{A}=\left[\begin{array}{llll}
1 & 0 & 0 & 1 \\
0 & 1 & 0 & 0 \\
0 & 0 & 0 & 1 \\
0 & 0 & 1 & -s
\end{array}\right], \quad U_{B}=\left[\begin{array}{llll}
1 & 0 & 0 & 0 \\
0 & 1 & 0 & t-1 \\
0 & 0 & 1 & 0 \\
0 & 1 & -s & 1
\end{array}\right]
$$

Then $A_{U}=\left(a_{i j}\right)=A\left[E^{-1}\right]\left[U_{1}^{T}\right]$ and $B_{U}=\left(b_{i j}\right)=B[U]$ are Minkowski reduced. Hence the points $A, A\left[E^{-1}\right], A\left[E_{2}\right], B, B\left[E_{1}\right], B\left[E_{2}\right]$ are extremal, and they are the vertices of the hexagon $R=L_{P} \cap K(w)$.

Thus, for any $\alpha, R$ is a fundamental domain of $\left\langle E, E_{2}\right\rangle$, and $\mathbf{Z}_{F}^{\times} /\{ \pm 1\}=\langle\epsilon, \eta\rangle$.

\section{REFERENCES}

[1] A. Ash, D. Mumford, M. Rapoport, and Y. Tai, Smooth Compactification of Locally Symmetric Varieties, Math. Sci. Press, Brookline, Mass., 1975. MR 56:15642

[2] A. J. Brentjes, Multi-dimensional continued fraction algorithms, Mathematical Centre Tracts 145, Mathematisch Centrum, Amsterdam, 1981. MR 83b:10038

[3] Bryuno, A.D. and Parusnikov, V.I., Klein polyhedra for two Davenport cubic forms, (Russian) Mat. Zametki, 56 (1994) 9-27; translation in Math. Notes 56 (1994) 994-1007 (1995). MR 96a:11061

[4] J.W.S. Cassels, An Introduction to the Geometry of Numbers, Springer-Verlag, 1959. MR 28:1175

[5] J.W.S. Cassels, Rational Quadratic Forms, Ac. Press, NY, 1978. MR 80m:10019

[6] H. Cohen, A Course in Computational Algebraic Number Theory, Graduate Texts in Math. 138, Springer-Verlag, 1993. MR 94i:11105

[7] H. Cohen, Advanced Topics in Computational Algebraic Number Theory, Graduate Texts in Math. 193, Springer-Verlag, 1999. MR 2000k:11144

[8] J. H. Conway and N. J. A. Sloane, Sphere packings, lattices and groups, Springer-Verlag, New York and Berlin, 1988. MR 89a:11067

[9] H. Davenport, On the product of three homogeneous linear forms. I, Proc. London Math. Soc., 44 (1938), 412-431.

[10] H. Davenport, Note on the product of three homogeneous linear forms, J. London Math. Soc., 16 (1941), 98-101. MR 3:70f

[11] H. Davenport, On the product of three homogeneous linear forms. IV, Math. Proc. Camb. Phil.. Soc., 39 (1943), 1-21. MR 4:212a

[12] H. Davenport, C. A. Rogers, Diophantine inequalities with an infinity of solutions, Philos. Trans. Roy. Soc. London, 242 (1950), 311-344. MR 12:394b

[13] P. B. Eberlein, Geometry of Nonpositively Curved Manifolds, The University of Chicago Press, 1996. MR 98h:53002

[14] Ennola, V., Cubic number fields with exceptional units, Computational number theory (Debrecen,1989), 103-128, de Gruyter, Berlin, 1991. MR 93e:11131

[15] M.-N. Gras, Table numérique du nombre de classes et des unités des extensions cycliques de degré de Q, Publ. Math. Fac. Sci. Besançon, fasc. 2 (1977/78). MR 52:10678 
[16] D. Grenier, Fundamental domains for the general linear group, Pacific J. Math. 132 (1988), 293-317. MR 89d:11055

[17] P. M. Gruber and C. G. Lekkerkerker, Geometry of Numbers, 2nd ed. North-Holland Publishing Co., Amsterdam, 1987. MR 88j:11034

[18] A. Korkine and G. Zolotarev, Sur les formes quadratiques, Math. Ann. 6 (1873), 366-389.

[19] G. A. Margulis, Indefinite quadratic forms and unipotent flows on homogeneous spaces, $C$. R. Acad. Sci. Paris Sér 1 Math. 304 (1987), 251-257. MR 88f:11027

[20] G. Niklasch and N.P. Smart, Exceptional units in a family of quartic number fields, Math. Comp. 67 (1998), 759-772. MR 98g:11125

[21] Minemura, Kenji, On totally real cubic fields whose unit groups are of type $\{\theta+r, \theta+s\}$, Proc. Japan Acad. Ser. A Math. Sci. 74 (1998), 156-159. MR 99m:11126

[22] Wladislaw Narkiewicz, Elementary and Analytic Theory of Algebraic Numbers, SpringerVerlag, Berlin and New York, 1990. MR 91h:11107

[23] Parusnikov, V.I., Klein polyhedra for complete decomposable forms, Number theory (Eger, 1996), 453-463, de Gruyter, Berlin, 1998. MR 99f: 11083

[24] S. S. Ryshkov and E. P. Baranovskii, Classical methods in the theory of lattice packings, Russian Math. Surveys, 34 (1979), 1-68. MR 81a:10045

[25] D. Shanks, The simplest cubic fields, Math. Comp. 28 (1974), 1137-1152. MR 50:4537

[26] Swinnerton-Dyer, H.P.F., On the product of three homogeneous linear forms, Acta Arith. 18 (1971), 371-385. MR 45:1844

[27] A. Terras, Harmonic analysis on symmetric spaces and applications. II, Springer-Verlag, Berlin and New York, 1988. MR 89k:22017

[28] G. Voronoi, On a generalization of the Algorithm of Continued Fractions, Doctoral dissertation, Warsaw, 1896. (=Collected works, vol. 1, Izdat. Akad. Nauk Ukrain. SSR, Kiev 1952, 197-391) (Russian). MR 16:2d

[29] L.Ya. Vulakh, The Markov spectrum for triangle groups, J. Number Theory, 67 (1997), 11-28. MR 99e:11093

[30] L.Ya. Vulakh, Farey polytopes and continued fractions associated with discrete hyperbolic groups, Trans. Amer. Math. Soc., 351 (1999), 2295-2323. MR 99i:11054

[31] L.Ya. Vulakh, Diophantine approximation in Euclidean spaces, CRM Proceedings and Lecture Notes, 19 (1999), 341-351. MR 2000j:11099

[32] L.Ya. Vulakh, The Markov spectrum for Fuchsian groups, Trans. Amer. Math. Soc., 352 (2000), 4067-4094. MR 2000m:11056

[33] L.Ya. Vulakh, Continued fractions associated with $\mathrm{SL}_{3}(\mathbf{Z})$ and units in complex cubic fields, Canad. J. Math. 54 (2002), 1305-1318.

[34] L. C. Washington, Class numbers of the simplest cubic fields, Math. Comp. 48 (1987), 371384. MR 88a:11107

[35] L. C. Washington, A family of cyclic quartic fields arising from modular curves, Math. Comp. 57 (1991), 763-775. MR 92a:11120

Department of Mathematics, The Cooper Union, 51 Astor Place, New York, New YORK 10003

E-mail address: vulakh@cooper.edu 